\title{
Fermi Timing and Synchronization System
}

\author{
R. Wilcox, J. Staples, L. Doolittle, J. Byrd, A. Ratti (LBNL) \\ F. X. Kaertner, J. Kim, J. Chen, F. O. Ilday (MIT) \\ F. Ludwig, A. Winter (DESY) \\ M. Ferianis, M. Danailov, G. D'Auria (ELLETRA)
}

\section{Outline}

1. Introduction

2. Synchronization requirements

3. An integrated system approach

4. Synchronization technology development at MIT

5. Synchronization technology development at LBL

\section{Introduction}

The Fermi FEL will depend critically on precise timing of its RF, laser and diagnostic subsystems. The timing subsystem to coordinate these functions will need to reliably maintain sub-100fs synchronicity between distant points up to $300 \mathrm{~m}$ apart in the Fermi facility. The technology to do this is not commercially available, and has not been experimentally demonstrated in a working facility. Therefore, new technology must be developed to meet these needs. Two approaches have been researched by different groups working with the Fermi staff. At MIT, a pulse transmission scheme has been developed for synchronization of RF and laser devices. And at LBL, a CW transmission scheme has been developed for RF and laser synchronization. These respective schemes have advantages and disadvantages that will become better understood in coming years. This document presents the work done by both teams, and suggests a possible system design which integrates them both. The integrated system design provides an example of how choices can be made between the different approaches without significantly changing the basic infrastructure of the system. Overall system issues common to any synchronization scheme are also discussed.

\section{General synchronization requirements}

Numerical modeling of the accelerator and FEL have resulted in synchronization requirements for the various subsystems, as listed in the following table.

\begin{tabular}{|l|l|l|}
\hline Subsystem & $\begin{array}{l}\text { Requirement, } \\
\text { fs RMS }\end{array}$ & Number of lines \\
\hline RF, S-band & 167 & 12 \\
\hline RF, X-band & 69 & 1 \\
\hline Photoinjector laser & 200 & 1 \\
\hline Seed laser & 100 & 1 \\
\hline Endstation laser & 100 & 2 \\
\hline Streak camera driver & 500 & 2 \\
\hline Streak camera fiducial & 100 & 2 \\
\hline Accelerator diagnostic & 100 & 6 \\
\hline
\end{tabular}

Table 2.1 Requirements for synch clients.

It is important to note that a timing signal generated at the synchronization system must travel through other devices to get to the point where the timing requirements have been specified, so that 
part of the error must be budgeted to the synchronized device and part to the synch distribution system itself. It is not possible to do this without making assumptions about the particular equipment to be used, so this allotment is only made for the specific systems described in later paragraphs.

Concerning the above table, one might ask "timing error with respect to what?" The requirements for the timing system are defined in terms of the differential timing error between the synchronization output being specified, and an output chosen as a reference. (We arbitrarily choose the seed laser timing line, as this is one of the most critical). Note that absolute, common mode timing errors slower than the time difference between lines are inconsequential, because the whole machine can time shift at once with no effect on performance. To be more specific when referring to different time scales of error, we break up the timing error into two ranges: DC (with an assumed period of 8 hours over which the spec must be maintained) to $10 \mathrm{~Hz}$ (initial machine reprate) is called "drift" and $10 \mathrm{~Hz}$ to modelocked laser Nyquist frequency (e.g. 40-50MHz) is called "jitter." This allows us to specify broad ranges of error, although a particular application might span a bandwidth that includes both drift and jitter. The technical significance of breaking the timing error bandwidth into ranges is that environmental perturbations are different (e.g. temperature versus acoustics), and the means of decreasing the errors are different (e.g. electromechanical control loops versus vibration damping).

\section{Integrated system design}

The integrated system approach is described below. A central clock system generates stable timing signals for the whole facility, sending them to locations up to $300 \mathrm{~m}$ away over optical fibers in a "star" configuration. Timing information is transmitted using either RF-modulated CW light (mainly for the Fermi RF systems) or pulsed light (mainly for laser systems). The overall design incorporates different synchronization methods, optimized for their particular application.

The synch system interfaces with the low-level RF electronics in the accelerator, with lasers in the photoinjector, seed and endstations, and with accelerator and x-ray diagnostics via precise timing signals. Also, a trigger for coarsely timed, low reprate devices throughout the facility is generated in synch with the precise timing signals, so that conventional delay generators can be used where needed.

Standard telecom fiber optical components at $1550 \mathrm{~nm}$ wavelength are used wherever possible, to enhance reliability. Controls are implemented digitally, to enable easy optimization as well as facilitate remote control and diagnostics. 


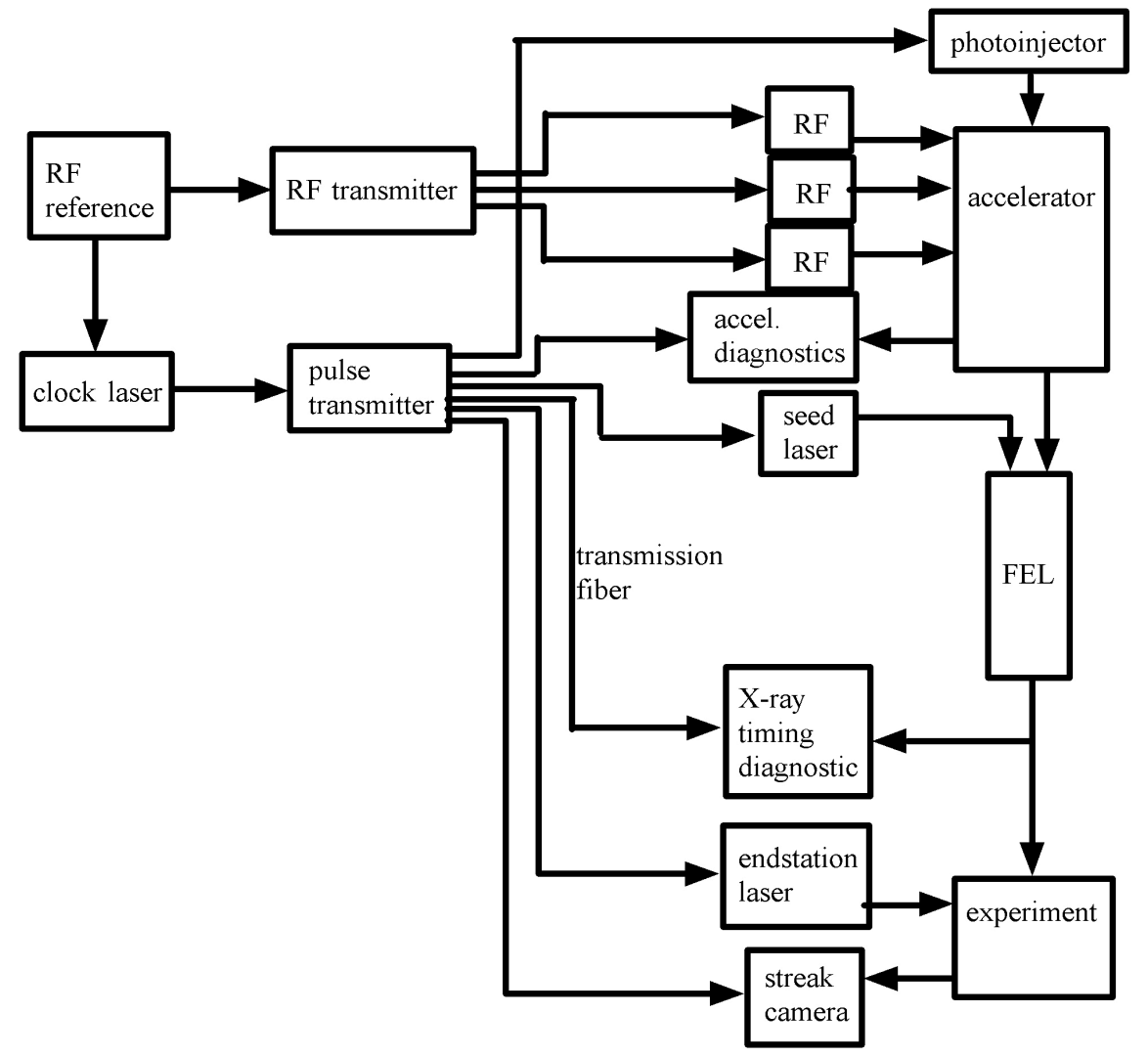

Figure 3.1. Overall block diagram of the integrated synch system.

\subsection{Integrated system requirements}

There are three types of precision synchronization clients in the Fermi system: RF generators, pulsed laser oscillators, and directly seeded laser amplifiers or timing diagnostics. These terms refer to the kind of application for the synchronization signal, as determined by a certain method of timing control. In the case of an application that uses RF directly, an RF signal is what is delivered. For pulsed laser oscillators, a laser pulse is delivered, and an optical method is used to synchronize the synched laser to the delivered pulses. In the case of direct seeding, optical pulses are delivered and used as the input to an optical system, most often an amplifier. It is a feature of the Fermi synch system that a variety of techniques can be used to synchronize different devices.

\subsubsection{RF requirements}

For an RF client (actually, the LLRF portion of the cavity driving electronics), the definition of the output of the synch system is an unamplified RF signal from a photodiode. (Note that in practice, the LLRF and synch systems will be integrated and optimized together) From studies of machine performance versus parameter errors, timing error budgets for the RF systems have been defined (ref. Gerardo's table). The smallest allowable timing error in the S-band sections is $167 \mathrm{fs}$ RMS at the cavity. The RF system itself is assumed to contribute half the error in quadrature error. Thus, if the errors of the synch and LLRF devices are uncorrelated, the requirement for the synch system is $118 \mathrm{fs}$. The requirement for the X-band linearizer is more stringent, at $69 \mathrm{fs}$ RMS. The LLRF contributes $49 \mathrm{fs}$ to this quadrature total, resulting in $49 \mathrm{fs}$ for the synch system. In order to preserve a comfortable margin of error, the timing error goals for the synch system will be $100 \mathrm{fs}$ for S-band and 50 fs for X-band RF delivery. 
The LLRF system will be able to average the RF signal received from the synch system over $1 \mathrm{~ms}$, so that noise above $1 \mathrm{kHz}$ is removed. Thus the noise spec for RF transmission at the receiver is from DC ( 8 hours) to $1 \mathrm{kHz}$ only.

\subsubsection{Laser synch requirements}

There are several pulsed laser oscillators in the Fermi facility. These include the photoinjector, the seed, endstation experimental lasers, and lasers used to drive streak cameras for diagnostic or experimental purposes. At present, these are being designed as systems with stand-alone modelocked oscillators, but some of them may be directly seeded if this technique is sufficiently developed. The definition of the synch system output in the case of synchronizing a modelocked laser oscillator is a baseband error signal to be delivered to the laser's piezo mirror high voltage amplifier. There is also an optical input to the synch system, to be given a portion of the oscillator's optical output.

Two laser applications that will not be included in the synch system requirements are the laser heater and a streak camera near the photoinjector. These will be serviced by portions of the optical pulse from the photoinjector laser, and thus synchronized by the same synch line as the photoinjector.

The synchronization requirements for these lasers is $100 \mathrm{fs}$ RMS, except for the photoinjector which is $200 \mathrm{fs}$, and the streak camera driver which is $500 \mathrm{fs}$. Each laser may be equipped with different devices for timing control (piezoelectrically actuated cavity mirrors with different mechanical resonances, for example), and thus contribute a different amount of timing error to the total. Wellcontrolled titanium sapphire oscillators have demonstrated less than $10 \mathrm{fs}$ jitter with respect to each other when locked using the proposed techniques (cross-correlation), so it is expected that the timing requirement can be met by optimized oscillators, and that the laser oscillator itself will contribute little to the total. There may however be long term drift introduced by subsequent amplifiers and other equipment, so until this characteristic has been measured, we will assume the total specification is split in equally between the timing and laser systems. Thus, out of $100 \mathrm{fs}$ for the seed and end station lasers, $70 \mathrm{fs}$ is allotted to the synch system itself. Out of $200 \mathrm{fs}$ for the photoinjector, $140 \mathrm{fs}$ is allowed for the photoinjector synch output. For a streak camera, the synch requirement would be $350 \mathrm{fs}$.

As with RF transmission, the receiving device (ultimately a modelocked laser) will have a limited response time and thus will be insensitive to high frequency jitter of the synch signal. Most lasers control repetition rate by moving a mirror with a piezoelectric actuator, limiting the response frequency to $1-10 \mathrm{kHz}$. The laser acts as a stable local oscillator in this case, responding only to the slower components of phase information from the synch system. Thus the jitter requirements for synch lines to modelocked lasers will cover the range from DC ( 8 hours) to the laser control bandwidth of about $1 \mathrm{kHz}$.

\subsubsection{Direct seeding requirements}

Direct seeding would be used to produce laser pulses when pulsed light is required at low power, and an oscillator is unnecessary or undesirable. The output of a direct seeding synch line is defined as a $1550 \mathrm{~nm}$ optical pulse with temporal and spectral characteristics adequate for injection into a subsequent optical system. Clients for direct seeding lines include bunch phase monitor diagnostics, streak camera fiducials, and electrooptic sampling bunch diagnostics, all of which are specified at $100 \mathrm{fs}$ RMS timing error. It is expected that a $1550 \mathrm{~nm}$ signal will be used in the electrooptic 
sampling and bunch phase monitor (which also works by electrooptic sampling) applications. A streak camera fiducial may need to provide a shorter wavelength to activate certain photocathodes, so the subsequent optical system may include an amplifier and frequency converters. These devices, as well as fiber optic transport of light signals in all cases, may introduce drift which contributes to the total timing error. Thus, without data on the synched systems, we can arbitrarily split the errors equally and allow $70 \mathrm{fs}$ to the synch system in the direct seeding case.

For direct seeding or time diagnostic applications, there is no integrator or stable local phase reference oscillator, so the full bandwidth of error in the synch system is transferred directly to the client. Thus the synchronization system requirement is from DC to half the pulse repetition rate (Nyquist frequency). This will not necessarily be the application's potentially lower reprate (e.g. $10 \mathrm{~Hz}$ in the case of timing diagnostics). Higher frequency errors will alias down to the sampling frequency, so high frequency errors must be controlled.

\subsubsection{Other requirements}

An additional, low-precision client for the synch system is the "slow" timing system that provides triggers to instrumentation, pulses klystrons etc.. This is derived from the master clock, and transmitted throughout the facility via a commercial digital or analog fiber link. Received timing signals trigger local delay generators with nanosecond precision.

To summarize the requirements as seen by this integrated system, the allotments of timing error to the synch system itself are shown in the table below.

\begin{tabular}{|l|l|l|}
\hline Subsystem & $\begin{array}{l}\text { Requirement, } \\
\text { fs RMS }\end{array}$ & Synch syst. allotment \\
\hline RF, S-band & 167 & $118 \mathrm{fs}$, DC- $1 \mathrm{kHz}$ \\
\hline RF, X-band & 69 & 49fs, DC-1kHz \\
\hline Photoinjector laser & 200 & $\begin{array}{l}140 \mathrm{fs}, \text { DC-loop BW } \\
(\sim 1 \mathrm{kHz})\end{array}$ \\
\hline Seed laser & 100 & $\begin{array}{l}70 \mathrm{fs}, \text { DC-loop BW } \\
(\sim 1 \mathrm{kHz})\end{array}$ \\
\hline Endstation laser & 100 & $\begin{array}{l}70 \mathrm{fs}, \mathrm{DC}-l o o p ~ B W \\
(\sim 1 \mathrm{kHz})\end{array}$ \\
\hline Streak camera driver & 500 & $\begin{array}{l}350 \mathrm{fs}, \text { DC-loop BW } \\
(\sim 1 \mathrm{kHz})\end{array}$ \\
\hline Streak camera fiducial & 100 & $70 \mathrm{fs}$, DC-50MHz \\
\hline Accelerator diagnostic & 100 & $70 \mathrm{fs}$, DC-50MHz \\
\hline
\end{tabular}

Table 3.1. Distribution of synch system requirements.

In most cases, the distribution of timing error between the synchronization system and the rest of the synchronized subsystem has been to split it equally, assuming uncorrelated noise sources adding in quadrature. To correctly specify the timing requirements one also must specify a bandwidth over which the timing noise is measured. The RMS number is an integration of a noise spectrum over this bandwidth. The allotment to the synch system must make assumptions about how the client system will react to timing noise. For instance, the LLRF system in the RF client will be able to average the incoming synch signal over $1 \mathrm{~ms}$, between shots occurring at $100 \mathrm{~ms}$ period. Thus the specified noise bandwidth of the synch signal can stop at $1 \mathrm{kHz}$, requiring the synch system to only be stable over longer time periods. Similarly, most laser synchronization systems have an effective loop bandwidth of around $1 \mathrm{kHz}$, limited by the speed of response of a moving laser cavity mirror. 
Thus it has been assumed that the lasers in Fermi will work similarly. When actual frequency modulation transfer functions are obtained, a more precise allotment of jitter budget can be made.

\subsection{Integrated system description}

Each synchronization signal starts from the master clock source, is transported over a delaystabilized fiber link, and is received by some type of synch receiver. The clock and methods of synchronization are described below.

\subsubsection{Clock}

Currently the highest stability clocks are those which use a combination of modelocked lasers and $\mathrm{RF}$ oscillators. The proposed master clock is a modelocked fiber laser with repetition rate stabilized to an RF source. Such lasers have demonstrated $\sim 10 \mathrm{fs}$ of jitter from $1 \mathrm{kHz}$ to $40 \mathrm{MHz}$, and are commercially available. The laser repetition rate is chosen so that it is a submultiple of the S-band RF frequency, such that a stable RF signal can be derived either from a multiple of the RF reference or by detecting a high harmonic of the laser reprate from its pulse train. By multiplication and division, other frequencies required by the facility can be generated, including the 10 or $50 \mathrm{~Hz}$ basic repetition rate. It is assumed that the existing $10 \mathrm{~Hz}$ timing system will be used, and will receive from the precision timing system a high frequency to be divided, which would be a submultiple of the S-band and X-band frequencies.

If an $\mathrm{RF}$ oscillator is provided at $491.64 \mathrm{MHz}$, this can be multiplied and divided to produce $2998 \mathrm{MHz}$ (S-band), $94.674 \mathrm{MHz}$ (laser reprate), $50 \mathrm{~Hz}$ (machine reprate), and $11424 \mathrm{MHz}$ (X-band frequency). Good RF oscillators have demonstrated around $10 \mathrm{fs}$ integrated jitter at error frequencies above $1 \mathrm{kHz}$, so there will be little differential jitter between synch lines due to clock jitter and differences in phase delay between the lines.

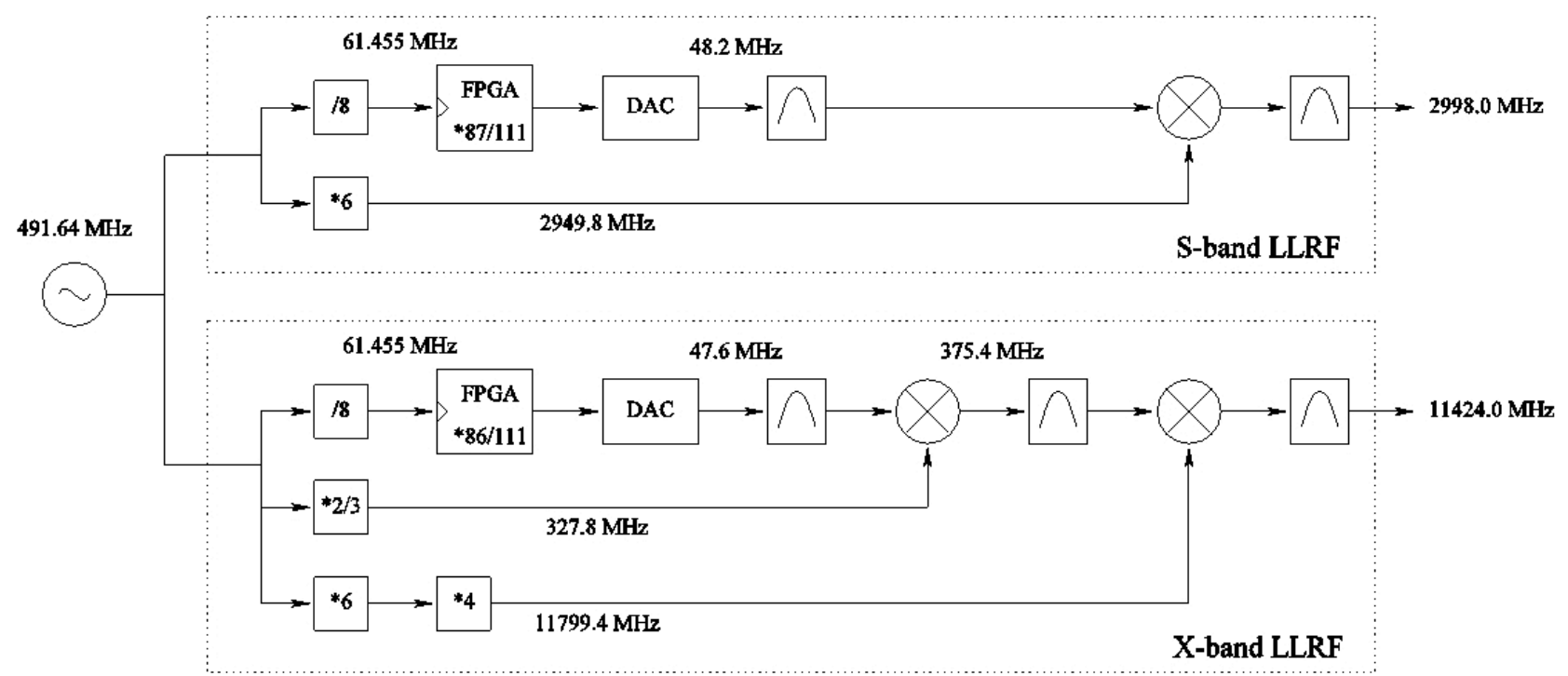

Figure 3.2. RF synthesizer chassis.

The goal of synchronizing the machine is to ensure timing synchronicity between all signals arriving at an experiment. Thus the time delay should be the same for all lines from the clock to a supposed pump/probe experiment. This may be true for lines of all the same type (such as all the RF lines to cavity drivers), but since there are synched devices of different types, it cannot be guaranteed that clock jitter will be common mode for all frequencies of timing error. That is, for one timing error frequency the effect of an RF signal on the final X-ray pulse and the effect of the 
photoinjector laser on that pulse may be synchronized at the experiment, but this may not be the case for another error frequency, due to the difference in phase modulation transfer function between the devices. As the relative phase delay may be larger for higher frequencies, it is important to have a clock with little integrated jitter at high offset frequency from the carrier.

\subsubsection{RF transmission}

In order to transmit RF synch signals, frequencies from the RF clock system are sent to an amplitude modulator. A $1530 \mathrm{~nm} \mathrm{CW}$ laser signal is modulated with the RF, and sent via fiber to a remote photodiode. To drive multiple lines, a fiber amplifier and splitter array are used, providing 16 outputs. The number of outputs can be increased at a later time by adding amplifiers and splitters. Since the frequency of the RF signal is arbitrary, any frequency can be transmitted with this scheme. The transmitted frequency need not be a harmonic of the clock laser repetition rate. This becomes important when two frequencies with a low common submultiple are used, as with the 2998 and $11424 \mathrm{MHz}$ S and X-band frequencies used in Fermi.

The split to multiple lines is maintained in a temperature controlled enclosure, to within 0.01 degree. This ensures that a $1 \mathrm{~m}$ long fiber will drift in delay by less than $0.4 \mathrm{fs}$. There will be several meters of fiber in the splitter array, depending on how it is implemented. A directional coupler array is lowest loss, but longer in total length (about $5 \mathrm{~m}$ ) than an optical chip waveguide tree splitter (about $2 \mathrm{~m}$ ). What is most important is that the differential delay between lines is constant, which requires the arms of the splitter tree are at the same temperature regardless of overall thermal drifts.

At the transmitter end, an interferometer measures the phase delay through the fiber and stabilizes the fiber length. As this is a phase shifting interferometer, the receiver end has a frequency shifter which is double passed by the retroreflected signal, so that when the retro signal and a reference wave interfere, a $94 \mathrm{MHz}$ beat is generated. A $47 \mathrm{MHz}$ reference frequency is transmitted along with the $3 \mathrm{GHz}$ to drive the frequency shifter. This signal is sent at $1310 \mathrm{~nm}$ wavelength to make it easy to separate with a wavelength division multiplexer (WDM). 


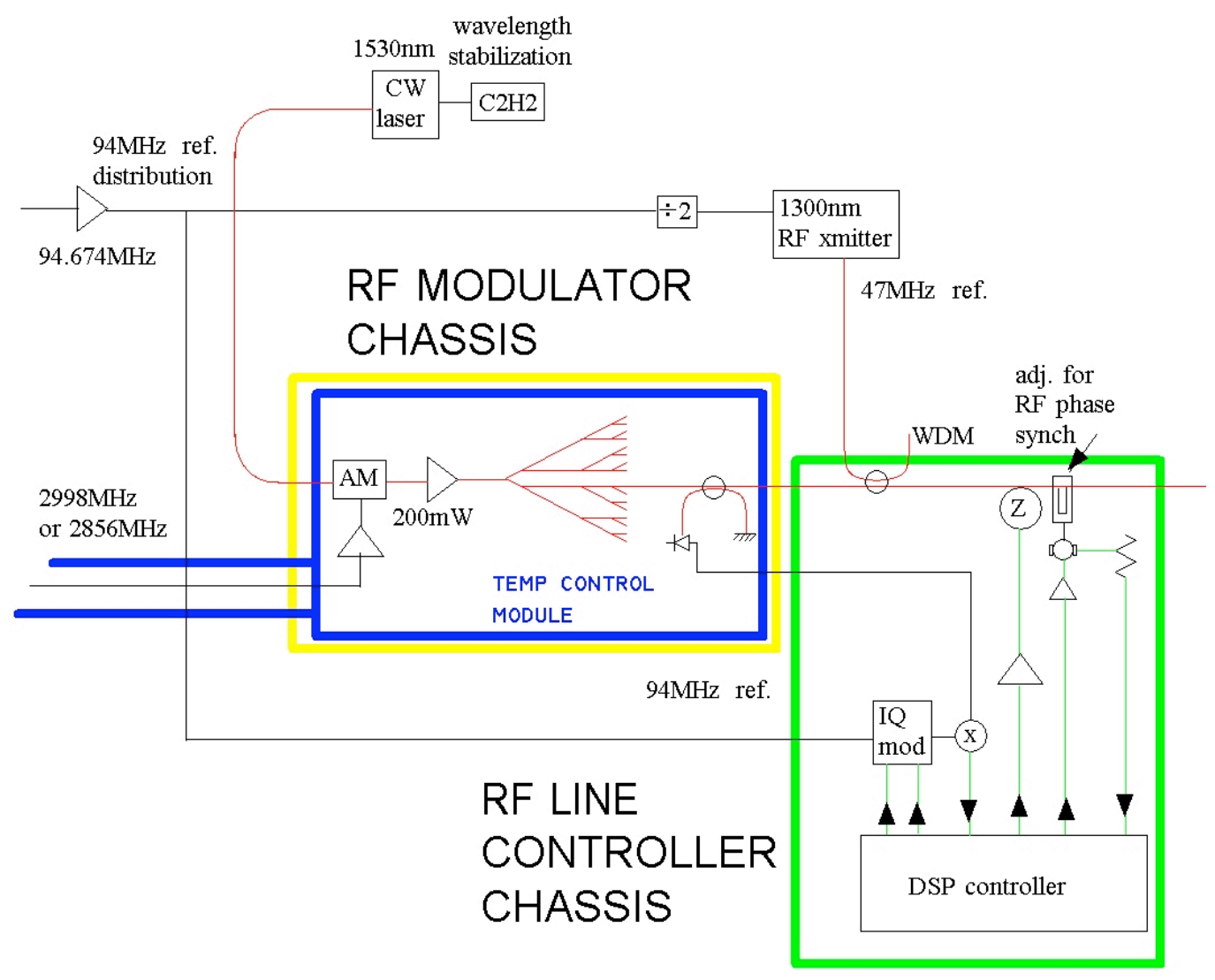

Figure 3.3. RF synch transmitter.

The fiber delay is controlled with a piezoelectric stretcher for high frequencies and a motorized delay for low frequencies. For a 300m fiber, a temperature change of 1 degree induces an $11 \mathrm{ps}$ change in delay. Thus the total delay range must be tens of picoseconds if the fiber temperature is held to within a few degrees. The mechanical delay also serves to synchronize the modulation of the signals at the interferometer detector. To obtain maximum signal, the peaks of the amplitude modulation of the local reference and the retroreflected signal must coincide at the photodiode. For a $3 \mathrm{GHz}$ transmitted frequency, the required delay range of pi phase is $167 \mathrm{ps}$. Assuming the fiber is held to $+/-2$ degrees, the additional delay will be $44 \mathrm{ps}$, for $211 \mathrm{ps}$ total. Commercial delays have ranges of 300 ps or more, so they can accommodate this delay.

At the receiver, the optical signal is received by a photodiode. The power on this diode must be regulated to within about $5 \%$ to maintain phase delay through the diode, due to variation of the junction capacitance with optical power. The average power is detected by the LLRF controller and used to feed back to the fiber frequency shifter RF amplitude. This forms an acousto-optic regulator. 


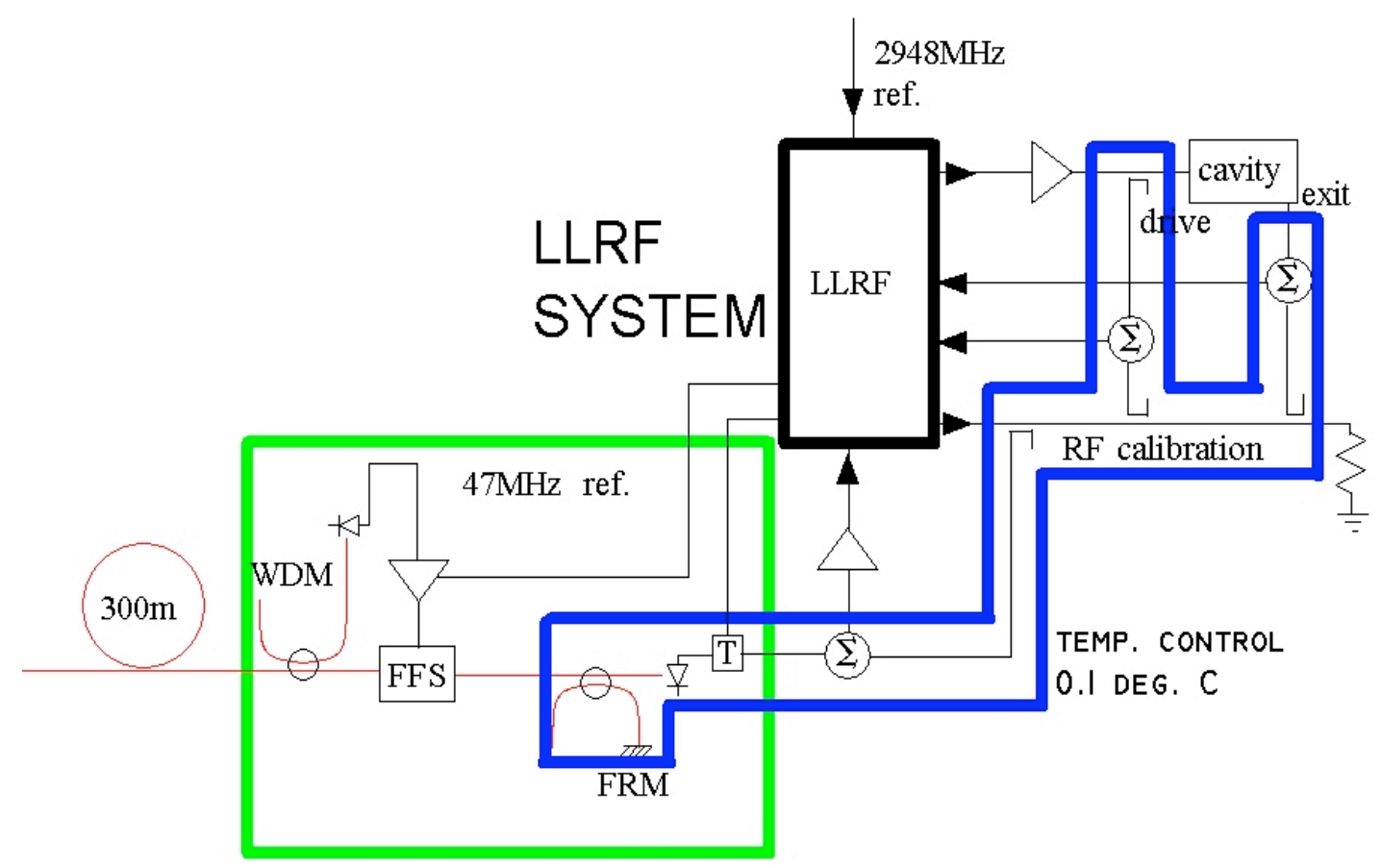

Figure 3.4. RF synch receiver.

\subsubsection{Short pulse oscillators}

In the case of synchronizing short pulse lasers, short ( 100fs) optical pulses will be transmitted from the optical clock, to receivers which optically cross-correlate the transmitted pulses with pulses from the laser to be synchronized. The cross correlation scheme mixes two optical signals in a nonlinear crystal, and detects the power of the mix product (the optical sum frequency) on a photodetector. The two pulses are partially overlapped, resulting in an output signal that is a sensitive function of the relative timing of the two pulses. The cross-correlator output becomes the timing error signal, to be fed back to a rate control on the receiver laser.

Since the sum frequency power is a function of the input optical pulse powers, amplitude noise on the input signal can cause noise on the sum frequency output, resulting in errors in synchronization. This sensitivity can be eliminated by implementing a balanced cross-correlator, where two crosscorrelations are performed. A pulse from one laser is split into two pulses separated in time by about one pulse duration. The pulse from the other laser is also split into two, both timed so that they sit temporally between the two pulses from the first laser, partially overlapping both of them. The pulses from the two lasers are mixed in two crystals, and the sum is detected by two detectors whose signals are then subtracted to form a differential error signal. Normally, the lasers are adjusted in relative timing so that the differential signal is zero. Thus, if one laser changes timing with respect to the other, the two mixing products from the two correlations will change differentially. If either laser changes power, the signal appears as common mode and is rejected by the signal subtraction. The system is therefore immune to amplitude noise. Cross-correlators of this kind have experimentally shown timing sensitivity less than $1 \mathrm{fs}$. Cross-correlation in general is the "gold standard" to determine relative timing of pulsed lasers.

Most lasers to be synchronized in FERMI are Ti:sapphire based systems operating at $800 \mathrm{~nm}$. The pulse train leaving the optical fiber link will be centered at $1550 \mathrm{~nm}$ wavelength. At typical power levels of $10 \mathrm{~mW}$ each, optical cross correlation in $1 \mathrm{~mm}$ thick BBO or LBO crystals can be performed. With 100 fs pulses conversion efficiencies of $>1 \%$ can be achieved. The 100 _W of blue 
light can easily be detected with standard silicon detectors. In fact, 1_W of power would be enough for the synchronization task.

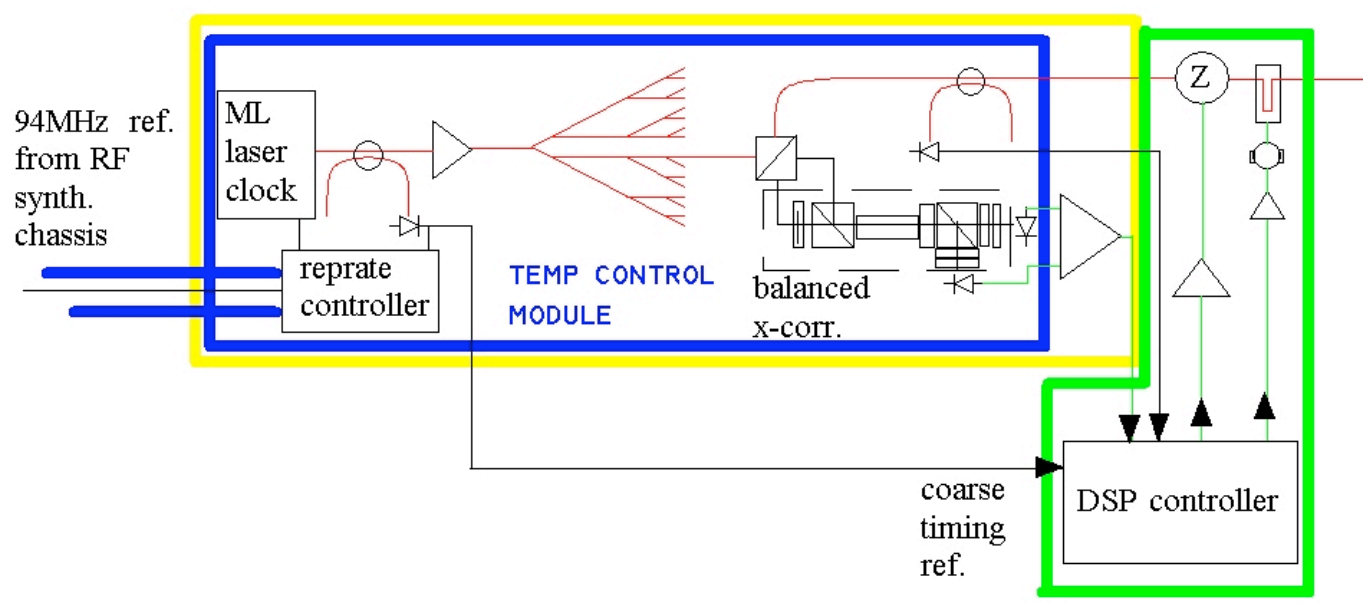

Figure 3.5. Pulse laser synch transmitter.

The transmission fiber has unknown birefringence which randomly varies over thermal and acoustic time scales, driven by temperature and mechanical perturbations. In a quiet environment, slow thermal variations are the most significant. Sum frequency mixing in the cross-correlator requires stable polarization of the two input optical signals, so an active polarization controller will be used at the end of the fiber. These are commercially available with around $1 \mathrm{kHz}$ bandwidth, and can maintain linear polarization to about $2 \%$ extinction, which is sufficient for the cross-correlator to work.

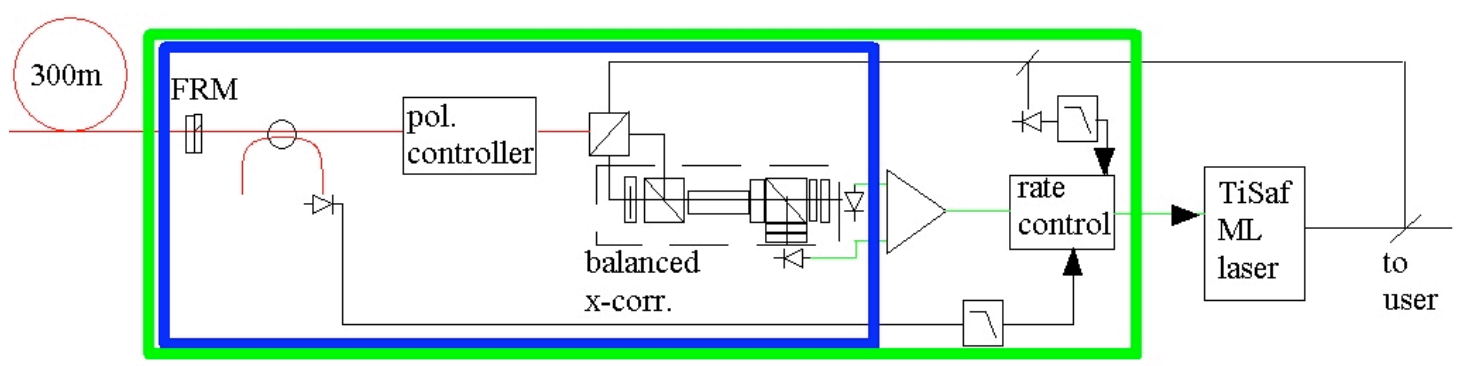

Figure 3.6. Pulse laser synch receiver.

Pulses transmitted through fiber experience dispersion and self-phase modulation (SPM) in the silica medium, as well as stimulated Raman scattering (SRS) at high intensities. The powers used will be below the SRS threshold, but dispersion and SPM will still occur. SPM does not significantly change the pulse envelope by itself, but will exacerbate dispersion by broadening the optical spectrum. Both of these effects can be minimized by using dispersion-managed fiber links. In this link, two kinds of fiber are used: normal dispersion fiber and dispersion shifted fiber (DSF), which has an opposite dispersion parameter. The two types of fiber are spliced together alternately, so that an optical pulse is alternately stretched and compressed. This has been found to be better than allowing the pulse to disperse in a long section of fiber then compensate with DSF at the end. Numerical simulations have shown that $100 \mathrm{fs}$ pulses can be transmitted over more than $300 \mathrm{~m}$ in such fiber links. 
Stabilization of the transport fiber time delay is accomplished similarly to the synchronization of the two lasers. A portion of the transmitted pulse is retroreflected back through the transport fiber to the transmitting end. The retroreflector is a Faraday rotator mirror, which ensures that the polarization of the light that gets back to the transmitter has polarization opposite from the light that was transmitted. Thus the transmitted and reflected signals can be separated by a polarizing beamsplitter, and the retroreflected signal has a stable polarization even though the birefringence of the transmission fiber is random and constantly changing. The retroreflected pulse (again maintained as a short pulse by the dispersion-managed fiber) enters a balanced cross-correlator along with a local reference pulse split from the transmitted pulse train. The cross correlator measures variations in the roundtrip group delay through the fiber compared with a local reference, resulting in an error signal that is used to stabilize the fiber length. The error signal drives a piezo fiber spool and a motorized delay, the same as in the interferometer scheme described above, so that fast short range errors and slow long range errors are dealt with separately. If the temperature of the transmission fiber is maintained to within $5 \Lambda \mathrm{C}$, the range of a several $\mathrm{mm}$ mechanical delay is sufficient to track thermally induced variations.

\subsubsection{Direct seeding and time diagnostics}

In order to avoid the expense, bulk and reliability issues with modelocked oscillators, some applications can use the transmitted optical pulses directly or after amplification. Pulses transmitted through fibers may not be as short or as "clean" as those from an oscillator, but not all applications are equally sensitive to pulse parameters. For diagnostics using electrooptic sampling, such as bunch shape detectors and arrival time monitors, low energy $100 \mathrm{fs}$ pulses are sufficient. Several diagnostics of this kind on the accelerator will use telecom-type electrooptic modulators, which are easy to use with the $1550 \mathrm{~nm}$ pulse trains. Delivery would be through cross-correlation delaystabilized fibers, providing a stable time reference. Variable optical delays at the receiving end can be set (for arrival time sensing) or scanned (for bunch shape measurement). If uncompensated higher order dispersion in the transmission fiber results in "wings" that reduce the dynamic range of the sampling measurement, nonlinear polarization rotation or nonlinear directional couplers could be employed to pass only the high intensity part of the pulse.

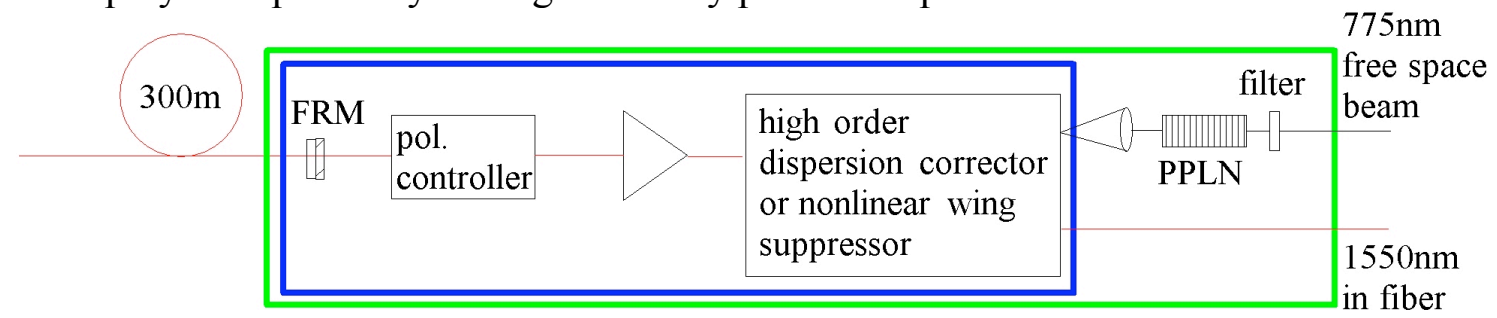

Figure 3.7. Direct seeding or time diagnostics receiver.

Since $1550 \mathrm{~nm}$ pulses can be amplified and frequency doubled to $775 \mathrm{~nm}$, these pulses can also be amplified in a titanium sapphire amplifier, and substitute for a titanium sapphire oscillator. If this application required a wavelength closer to the peak of titanium sapphire gain, the modelocked fiber clock laser could be built to emit in the telecom "L-band" near $1600 \mathrm{~nm}$, which would double to $800 \mathrm{~nm}$. Frequency doubling cleans up pulse wings due to nonlinear power conversion, although there is some bandwidth narrowing resulting in a longer pulse duration. Frequency converted pulses can be used as streak camera fiducial signals, and as photoconductive switch drivers after amplification in a regenerative amplifier. For instance, after being amplified in a $200 \mathrm{~mW}$ fiber amplifier and frequency converted, the $775 \mathrm{~nm}$ pulses will have about $1 \mathrm{~nJ}$ of energy. This compares well with the $3-5 \mathrm{~nJ}$ available from a titanium sapphire oscillator, and can be easily amplified in a regen up to the milliJoule level. 


\subsection{Integration, controls and diagnostics}

Since all of the synchronization methods described above are coordinated from the same master clock laser stabilized to an RF reference source, the different types of signals will be synchronized to each other, and devices synched to these signals will be synched to all the other devices in the Fermi machine. Thus, it doesn't matter which type of synchronization signal is used in a particular application, as global timing stability is guaranteed.

With all the synchronized devices located in different parts of the machine, checking that they are all correctly timed will be a difficult exercise, requiring some dedicated period of operation of the whole system. A less difficult task will be to verify that the synchronization lines all work to their respective specifications. It is not sufficient to measure the variation of the control loop error signals, as this is only an "in-loop" measurement and cannot ensure the loop itself is working. Thus we need a second device temporarily located near the synchronized device, to do a comparison between two lines. It is expected that each location will be served by at least one redundant fiber, so that a fiber break can be bypassed. This second fiber can be connected to an extra stabilizer at the transmitter end, reserved for this purpose (or as a spare). A device similar to that at the receiver end is brought in to the same location, and compared. For instance, an RF line can be checked by comparing the output of its LLRF controller with a similar controller receiving RF from the second line, or simply by using the first LLRF controller to compare two RF signals (which is what it is designed to do). It is unlikely that they will both vary together if one of the lines is not properly functioning (though this is not impossible). Similarly, laser synchronization can be checked by bringing around a relatively portable and inexpensive fiber modelocked laser, and cross correlating the output of it and the laser to be checked. The test laser will have been previously verified to be robust and reliable in synchronization tests, serving as a sort of measurement instrument, not unlike the local oscillator in a spectrum analyzer.

Control and diagnostics of the synchronization system will be accomplished by communicating with the digital FPGA controllers that run all the compensation loops. These are similar to those used in the LLRF controllers presently. These devices can monitor the signal levels, error signal history, percent of dynamic range used, and other parameters of interest. All data can be logged and monitored automatically, to predict problems. Typical operation of the synch system control loops may involve opening the loop, adjusting some parameters, and closing the loop again. This can be done through the FPGA. Some adjustments may be to optimize the loop gain parameters, which can be done easily by programming the controller. The major task in this area will be to design the interface with the control system so that most housekeeping functions are performed automatically rather than by operator control.

Each user of a synchronization line will need to be able to locally vary parameters and loop functions, and monitor diagnostics such as input signal strength. This implies a GUI or some kind of software interface at the location of the synched device, lasers in particular. The laser user will send a part of their oscillator output to a port in the synch receiver, where it will enter the crosscorrelator. The receiver will have to reside on the optical table, since all time-critical signals inside the receiver are stabilized thermally and cannot be connected via unstabilized cables. The output from this box on the table will connect via electrical cable to the laser controls.

In some cases there may be several synchronization clients located in the same area, so that a local "fan out" from one synch receiver can be used by all. Local synch signals could be distributed on a temperature-controlled fiber, maintained at constant temperature by flowing water. If a fiber link 
was $5 \mathrm{~m}$ long, one degree $\mathrm{C}$ variation over the whole length would result in $175 \mathrm{fs}$ of delay change, which is outside the requirement for most applications. A watertight fiber enclosed in a water stream controlled to 0.1 degree $C$ would maintain optical delay within $17.5 \mathrm{fs}$, which is acceptable. A small thermoelectric chiller located at the synch receiver would service several temperature stabilized lines at low flow rate (to minimize turbulence). The water/fiber assemblies may be a slight variation on previously existing multiple-fiber cable designs, which can be quite elaborate.

The master clock and RF source should be located near the photoinjector. This allows for propagation of synchronized signals through the machine in parallel with the propagation of electron beams and x-rays, such that the signals meet at the experiment with a minimum of fiber delay. Propagation through fiber is at about $70 \%$ the speed of light, arguing for a location somewhat farther to the experiment end, though any location could be accommodated by adding fiber length. Minimizing fiber length minimizes the dynamic range required of the fiber stabilization loops, given a certain temperature stability of the fiber runs.

All equipment at the transmitting end will be housed in standard electronic equipment racks, including the master clock laser and $\mathrm{CW}$ laser. The room in which the racks reside should be well temperature controlled to within a few degrees, to minimize influence on the temperature stabilized parts of the system. Depending on the results of early experiments, the fibers in the long runs may have to be specially isolated from acoustic vibrations. This may only require the addition of foam padding to standard telecom fiber cable trays, or it may require some more elaborate solution. Good management of the fiber path should be maintained right up to the receiver chassis in every case.

It is important to minimize acoustic perturbations of the fiber as well as thermal variations, which means that the fiber should be routed through the linac and undulator tunnels. This may be a radiation environment, but measurements indicate radiation may not be a problem in actual operation. Gamma rays and neutrons can cause an increase in loss through Ge-doped fiber, but modern fibers are still relatively insensitive. Measurements must be made of the typical radiation environment in the tunnel, and fiber options evaluated. Radiation-hard fiber with no germanium in the core can be used with the $\mathrm{CW}$ transmitting links, but it may not be possible to obtain radiation hard dispersion compensating fiber for the dispersion-managed pulse transmitting links. The criterion for radiation darkening would be the acceptable transmission over a reasonable period between replacing fibers. Acceptable transmission is based on the power margin for the links, especially the length stabilization systems which see a double-passed fiber loss. As received power decreases, so does link reliability, since the probability of an out-of-spec timing error increases. This situation is similar to telecom measurements of bit error rate (BER), and will need to be carefully evaluated in terms of overall system reliability and "up time."

\section{Synchronization technology development at MIT}




\subsection{INTRODUCTION}

A conceptual schematic of the envisioned timing and synchronization system in the context of the FERMI XFEL, which is a fully seeded fourth-generation light source is depicted in Figure 1. An optical master oscillator (OMO) in the form of a mode-locked laser is tightly synchronized to the reference microwave oscillator (RMO), which times the overall facility. Very low noise microwave oscillators that may serve as a reference microwave oscillator with a superior timing jitter well below $10 \mathrm{fs}$ within the typical repetition rate of the RF-macro pulses are commercially available, for example sapphire loaded dielectric resonator oscillators [2]. The OMO, i.e. a mode-locked laser, generates a stream of very low jitter $100 \mathrm{fs}$ pulses that are distributed to the individual sub-systems that need tight synchronization to the microwave reference of the facility. The optical pulse train has to fulfill two tasks.

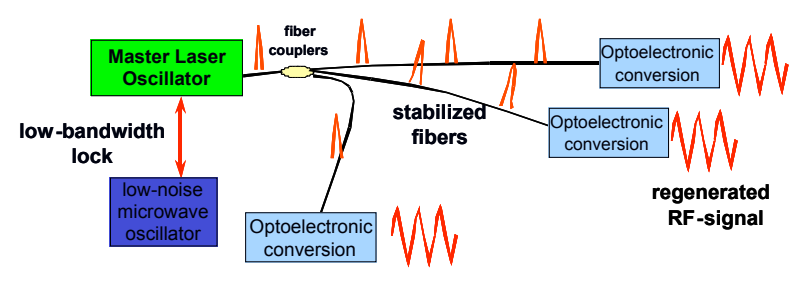

Figure 4.1: Conceptual schematic of the proposed optical synchronization system.

First, it serves as the means for stabilizing the group delay of the pulses in the fiber link to a multiple of the repetition time of the pulses with femtosecond precision. Second a fraction of the pulse train is coupled out from each end of the fiber links. The output pulse train at each fiber end can be used for synchronization of microwave or optical subsystems to the RMO.

In the following, we will discuss the requirements and hurdles that need to be overcome in implementation of the proposed overall timing distribution system.

\subsection{RF-EXTRACTION AND SYNCHRONIZATION}

RF Extraction essentially means to extract from the optical pulse stream leaving the fiber a highly stable and low jitter RF-signal either at the fundamental repetition rate of the pulse train or eventually one of its harmonics. RF-synchronization means for example to lock the OMO tightly to the RMO.

Upon photo-detection, the short optical pulses, of sub-picosecond length, are converted into a train of electronic pulses of typically $50-100$ ps length if a $10 \mathrm{GHz}$ detector is used or even longer depending on the detectors bandwidth, see Figure 4.2. The short optical $\begin{array}{llllll}\mathrm{p} & \mathrm{u} & \mathrm{l} & \mathrm{s} & \mathrm{e} & \mathrm{s}\end{array}$ 

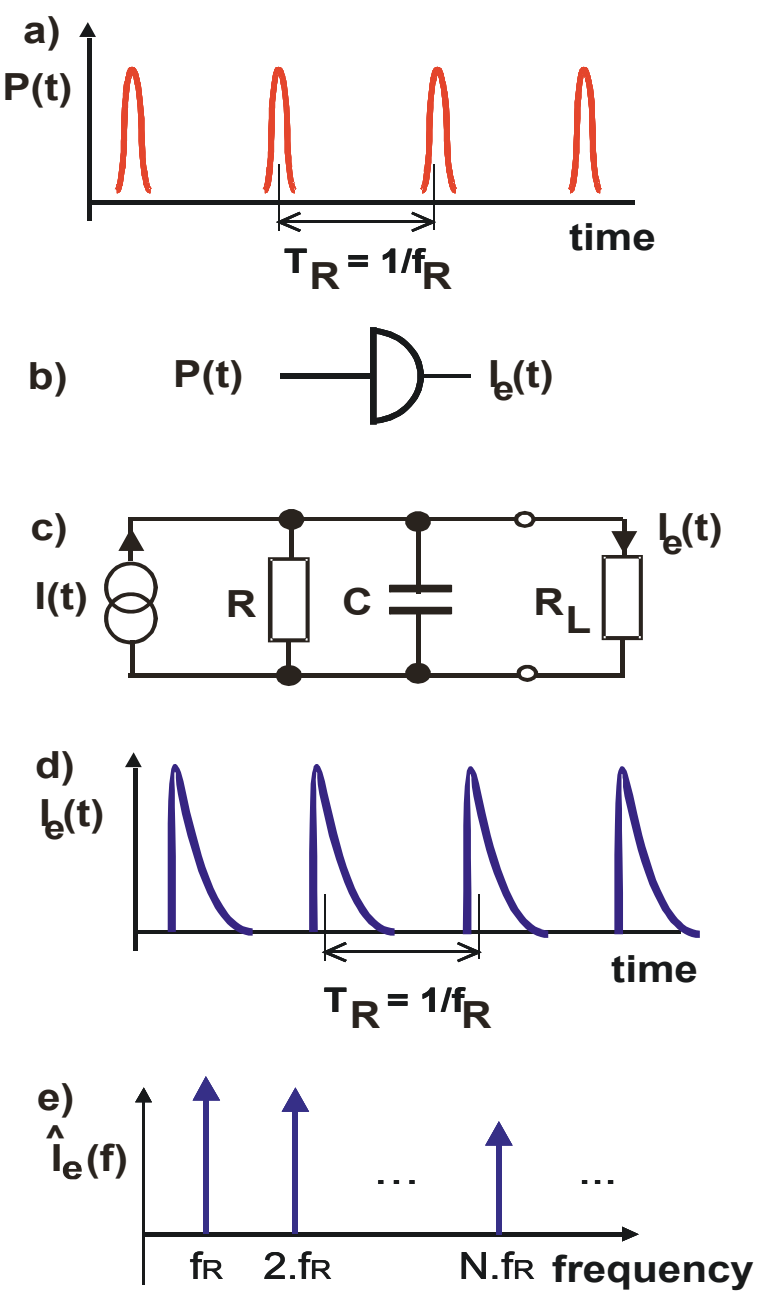

Figure 4.2: a) Optical pulse train with instantaneous power $\mathrm{P}(\mathrm{t})$; b) Photo detection with external current $\mathrm{I}_{\mathrm{e}}(\mathrm{t})$; c) Equivalent circuit of photo detector with external load; d) External current; e) Fourier components of external current.

eventually converted into more than a 1000 times longer electronic pulses $I_{e}(t)$ external to the photo detector. A reverse biased photo-detector can be modeled as a parallel RCcircuit driven by an ideal current source following precisely the optical intensity profile, $\mathrm{I}(\mathrm{t}) \sim \mathrm{P}(\mathrm{t})$, see Figure $4.2 \mathrm{~b}$ and $4.2 \mathrm{c}$. The capacitor models the space-charge capacitance of the pn-junction. The output pulse stream of the photo-detector is the convolution of the optical pulse train with the internal photo-detector impulse response. Or expressed differently, in the frequency domain all Fourier components $\hat{I}_{e}\left(\omega_{n}\right)$ of the periodic power variation in the optical pulse stream are multiplied by the frequency response of the photo-detector.

$$
H(\omega)=\frac{R_{L}}{R_{p}} \frac{1}{1-i \omega \tau}
$$

evaluated at each frequency component $\omega_{n}=2 \pi f_{n}=2 \pi n f_{r}$. Here, $R_{p}=\left(R / / R_{L}\right)$. Therefore, the different harmonics are phase shifted or experience a group delay 


$$
\tau_{n}=-\left.\frac{d}{d \omega} \arg (H(\omega))\right|_{\omega_{n}}=\frac{\tau}{1+\left(\omega_{n} \tau\right)^{2}} .
$$

This formula essentially tells us that all harmonics within the detector bandwidth simply see the mostly constant group delay due to the RC-lowpass filter. However, the space charge capacitance of the detector depends on the incoming optical intensity or pulse energy. Therefore, intensity fluctuations are converted to timing fluctuations of the detector output signals via the AM-to-PM conversion coefficient

$$
C_{R F}^{A M-P M}=\left.\frac{d \tau}{d P}\right|_{P_{0}}=\left.\tau \frac{1}{C} \frac{d C}{d P}\right|_{P_{0}}
$$

This simple model explains already one cause that might lead to an intensity to phase conversion due to photo detection as has been observed recently [4, 5]. Additional nonlinear effects during saturation of the diode in pulse operation may contribute to this effect and a more thorough investigation is necessary. We have measured this conversion coefficient with the setup shown in Figure 4.3 and results for $10 \mathrm{GHz}$ photo detectors from EOT are shown in the inset for a reverse bias voltage of $6 \mathrm{~V}$. Typical numbers for the conversion coefficient may range from $C_{R F}^{A M-P M}=1-5 \mathrm{ps} / \mathrm{mW}$ depending on bias conditions, which significantly change the space charge capacitance and the sensitivity of the capacitance to power fluctuations, which are also consistent with conversion factors stated in [5]. To obtain an RF output signal with about $80 \mathrm{~dB}$ SNR in a $100 \mathrm{kHz}$ bandwidth an optical power of about $5 \mathrm{~mW}$ is necessary on the photo detector. Then even at a relative intensity noise level of $0.1 \%$ AM-to-PM conversion results in a timing jitter ranging from 5-25 fs, which clearly can not be neglected for synchronization on the $10 \mathrm{fs}$ level. Thus long term stable RF-extraction from a pulsed optical source by direct photo detection is only possible, if the diode power on the detector is actively stabilized using the DC-output of the detector and a proper feedback loop.

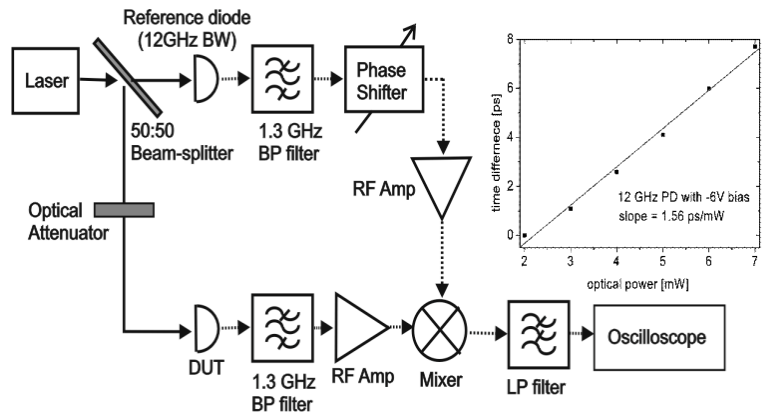

Figure 4.3: AM-to-PM conversion factor measurement setup and the result when the reverse bias is set to $6 \mathrm{~V}$. The measured slope is $1.56 \mathrm{ps} / \mathrm{mW}$.

Several ideas to achieve the stabilization of the optical power on the photodetector can be implemented. First one simply can use a feedback loop on a modulator in front of the diode. The DC-component of the current is monitored and stabilized via the modulator and a feedback loop.

An alternative way to overcome the AM-to-PM excess noise has been recently demonstrated [6] by implementing an optical-microwave phase detector that uses a 
timing transfer in the optical domain before detection. Recently, this component has been further developed to a fully fiber integrated optical-microwave phase detector as shown in Figure. 4.4. The balanced optical-microwave phase detector is based on precise phase detection by use of a differentially-biased Sagnac waveguide-loop and synchronous detection, which is similar to Sagnac-loop based gyroscopes [10] and clock recovery systems [11]. Figure 4.4 shows the conceptual diagram of the balanced optical-RF phase detector.

In Figure 4.4, the solid line and dotted line indicate optical beam path and electrical signal path, respectively. The optical beam path can be implemented either by an optical fiber or a high index contrast waveguide. Part of the input optical pulse train is tapped to the Photodiode \#1. This diode is used to generate a reference signal for synchronous detection. In principle, any frequency component up to half the repetition frequency $\left(f_{R} / 2\right)$ can be used as a reference signal. To maximize the signal level, $f_{R} / 2$ generated by a frequency divider (divide-by-two) is preferred for a reference signal. The reference signal is applied to both the phase modulator and the mixer.

The rest of the input pulse train is sent to the Sagnac-loop. By the phase modulation at the frequency of $f_{R} / 2$, the output pulse train from the Sagnac-loop has an amplitude modulation as shown in Figure 4.5. The amplitude of this signal is proportional to the phase error between the input optical pulse train and the input RF-signal. Therefore, we need to detect the amplitude of this modulated signal. After bandpass-filtering this signal at $\mathrm{f}_{\mathrm{R}} / 2$, we can mix this signal with the reference signal at the mixer. By this downconversion to the baseband, we can extract the phase error signal. Detailed mathematical derivation is shown in the Appendix.

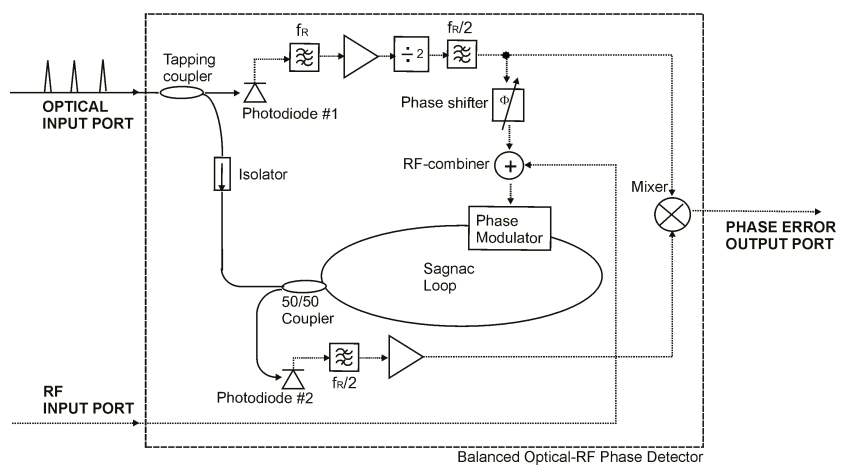

Fig. 4.4: Conceptual diagram of the balanced optical-RF phase detector. Photodiode \#1 is used to generate a synchronous detection reference signal with half the repetition frequency $(\mathrm{fR} / 2)$. Photodiode \#2 detects the output of the Sagnac-loop. The phase-error signal is encoded as the amplitude of $\mathrm{fR} / 2$ frequency component. The mixer down-converts the phase-error signal at $\mathrm{fR} / 2$ to the baseband by synchronous detection with the reference signal. This baseband signal is the phase error output of the balanced opticalRF phase detector. Solid line: optical beam path; dotted line: electric signal path.

When it is locked, the output from the phase detector is "balanced" since the amplitude noise from both optical and RF-signals is eliminated by synchronous detection. That is the reason why it is named as a balanced optical-RF phase detector. This scheme is also based on the timing information transfer to the optical domain - the timing error signal generates an amplitude modulation to the pulse train in the optical domain. Since it does not rely on the individual frequency component from direct photodetection, the AM-to-PM conversion does not affect the system. 


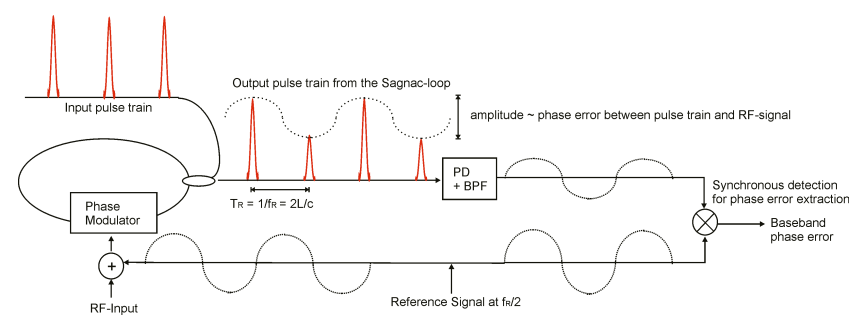

Figure 4.5: Schematic diagram of the synchronous detection between the amplitude-modulated pulse train and the reference signal. PD: photodetector; BPF: bandpass filter.

Another advantage of this phase detector is that its phase detection sensitivity is scalable to sub-femtosecond precision by increasing optical power and/or modulation depth of phase modulator. The phase detection sensitivity is proportional to the average optical power in the Sagnac-loop and the square of the modulation depth.

The noise performance is fundamentally limited by shot noise in the photodetection process. A phase noise floor set by shot noise can be derived and the final result is rather simple, $S_{\varphi, s h o t}=\frac{4 q}{R P_{\text {avg }} \Phi_{0}^{2}} \mathrm{rad}^{2} / \mathrm{Hz}$, where $\mathrm{q}(\mathrm{C})$ is the electron charge, $\mathrm{R}(\mathrm{A} / \mathrm{W})$ is the photodiode responsivity, $P_{\text {avg }}(\mathrm{W})$ is the average optical power in the Sagnac-loop, and $\Phi_{0}$ (rad) is the phase modulation depth from the VCO output. This result shows, again, the scalability of the achievable phase noise and timing jitter by increasing optical power and modulation depth. In reality, the system performance can be limited by thermal noise and other technical noise from the electronics. Therefore, it is very important to minimize the noise figures of the active components in the electronic domain for implementing a shot noise-limited system.

Figure 4.6 shows the experimental demonstration setup for the RF-signal regeneration from an optical pulse train by use of the balanced optical-RF phase detector.

All the optics parts in the phase detector were implemented with commercial 1550$\mathrm{nm}$ optical fibers. A stretched-pulse Er-doped fiber laser (repetition frequency $\mathrm{fR}=44.26$ $\mathrm{MHz}$, center wavelength $=1550 \mathrm{~nm}$ ) is used as the optical pulse source. Part of the input pulse train is tapped to the pin-PD \#1 to generate a synchronous detection reference signal with half the repetition rate $\left(\mathrm{fR}_{\mathrm{R}} / 2\right)$ of the optical pulse source. In parallel, a signal with the frequency $10.5 \mathrm{fR}$ is also generated to modulate the circulating pulse train in the fiber-loop. In fact, any frequency components of $(n+1 / 2) f$ can be used for this purpose. The $10.5 f_{R}$ (instead of $f R / 2$ ) is used to reduce the necessary fiber-loop length and the temperature sensitivity of the system.

The rest of the input pulse train is sent into the polarization-maintaining (PM) fiber Sagnac-loop with a phase modulator. The phase modulator is positioned in such a way that the optical delay between counter-propagating pulses at the phase modulator is set to $\Delta t_{\text {delay }}=1 /\left(2 \_10.5 \mathrm{fR}\right)$. This assures that the two circulating pulses experience opposite phase modulations. The VCO (PSI DRO-10.225 from Poseidon Scientific Instruments, Ltd.) has a center frequency of $10.225 \mathrm{GHz}$ which corresponds to the 231 st harmonic of the fundamental repetition rate. The pulse train detected at the fiber-loop output (pin-PD \#2) has an amplitude modulation with frequency $\mathrm{f}_{\mathrm{R}} / 2$ whose amplitude is proportional to the phase error between the pulse train and the VCO RF-signal (as shown in the previous 
section). The amplitude modulation is converted to the baseband phase error signal by synchronous detection with a mixer. This phase error signal is filtered and controls the low-noise VCO. Feeding back the output from VCO to the phase detector closes the locking loop. Long-term stable locking between the laser and the VCO over several hours is demonstrated.

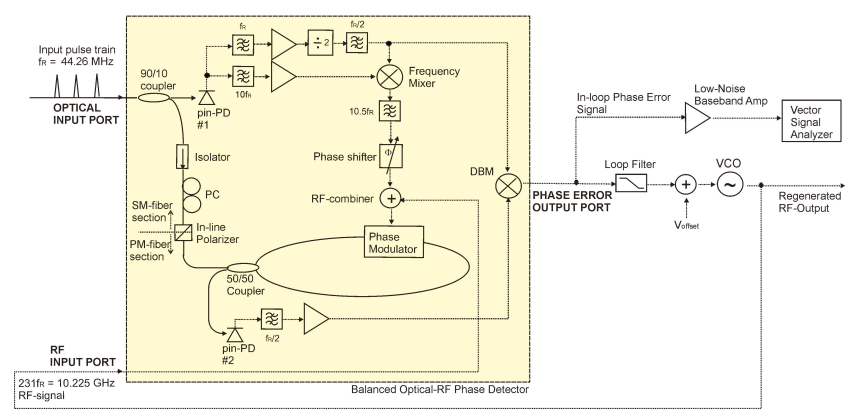

Figure 4.6: Schematic setup for RF-signal regeneration from an optical pulse train by use of the balanced optical-RF phase detector. DBM: double-balanced mixer; PC: polarization controller; PD: photodiode; PM: polarization-maintaining; SM: single-mode; VCO: voltage-controlled oscillator. Solid and dotted line indicate optical (in optical fiber) and electric signal paths, respectively.

Figure 4.7 shows the measured in-loop phase noise.

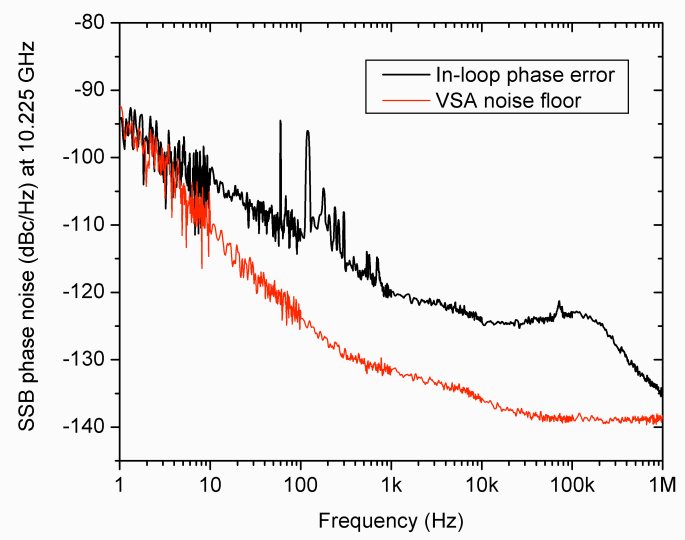

Figure 4.7: The single-sideband (SSB) in-loop phase noise spectra at $10.225 \mathrm{GHz}$. Black line: in-loop phase noise when it is locked. Red line: vector signal analyzer (VSA) noise floor. The integrated timing jitter from $1 \mathrm{~Hz}$ to $1 \mathrm{MHz}$ corresponds to $8.8 \mathrm{fs} \pm 2.6 \mathrm{fs}$

The voltage signal from the phase detector was measured with a low-noise vector signal analyzer (VSA), and converted into single-sideband (SSB) phase noise at $10.225 \mathrm{GHz}$. The black line shows the in-loop phase noise when it is locked. The red line is the noise floor from the VSA. This measurement shows that the integrated in-loop timing jitter is $8.8 \mathrm{fs} \pm 2.6$ fs from $1 \mathrm{~Hz}$ to $1 \mathrm{MHz}$. The stated error is determined by the VSA noise floor. Note that this is the first result, and we expect to improve the noise performance close to or below $1 \mathrm{fs}$ residual timing jitter. 


\subsection{TIMING STABILIZED FIBER LINKS}

Figure 4.8 shows in detail the envisioned fiber optic link. An Er-doped fiber or bulk laser with a repetition rate $f_{O M O}$ that is a sub multiple of the European S-band frequency $f_{S}=$ $2998 \mathrm{MHz}$. For example the $f_{O M O}=499.667 \mathrm{MHz}$, the sixth sub-harmonic seems to be a good choice, since one might still expect to build very low noise modelocked lasers at these medium high repetition rate, while the pulse energy is significantly decreased at a given average power in the pulse train to avoid nonlinearities in the fiber link.

Multiplication with 6 gives the S-band frequency, division by 10 relates to the machine repetition rate of $50 \mathrm{~Hz}$. The mode-locked laser itself is referenced to the microwave oscillator of the facility using for example the balanced optical-microwave phase detector discussed before. However, feedback is now given to the modelocked laser (OMO), rather than the RMO.

The output pulse train of the oscillator with an expected average power of about $10 \mathrm{~mW}$, will be amplified to $1 \mathrm{~W}$, so that up to 20 channels can be later supplied with up to $50 \mathrm{~mW}$ of power each. This pulse train is than sent into $\mathrm{N}$-channels, with $\mathrm{N}$ up to $\mathrm{N}=20$. Figure 8 shows at the moment only $N=2$. One of the two channels is then shown in detail. At the input of the fiber link will be a polarizing beam splitter cube, that is directly connected to fiber. These custom made components are sold by Oz-Optics. The input light is polarized at $45^{\circ}$. The output at the top is sent into the fiber link. To be able to adjust all fiber links later to equal length, that is then dynamically stabilized, we put via fiber collimators a free space section into the link. This section can also be motorized with nano-precision stages, that can easily take out all slow but large long term drifts in fiber length. Drifts up to several millimeters in length can be easily taken out. Then only a very course temperature stability of the fiber link needs to be maintained

Next, the pulse goes through a short fiber stretcher, which has a bandwidth of up to $10 \mathrm{kHz}$ for taking out the high frequency noise or short term fluctuations in the fiber. Then the $300 \mathrm{~m}$ long link is laid out in a dispersion-compensated fashion, which we will discuss in the next section. At the end of the link the pulse hits a Faraday rotator with $50 \%$ output coupling. The output can be used for synchronization purposes or as shown in Figure 4.8 to compare it with the output of the neighboring link via cross correlation. This enables also later diagnostics of the various links by comparing two of them against each other. Due to the Faraday mirror, the light is reflected into the opposite polarization on its return through the fiber link. The light reflected back to the beam splitter with orthogonal polarization states directly passes the polarizing beam splitter.

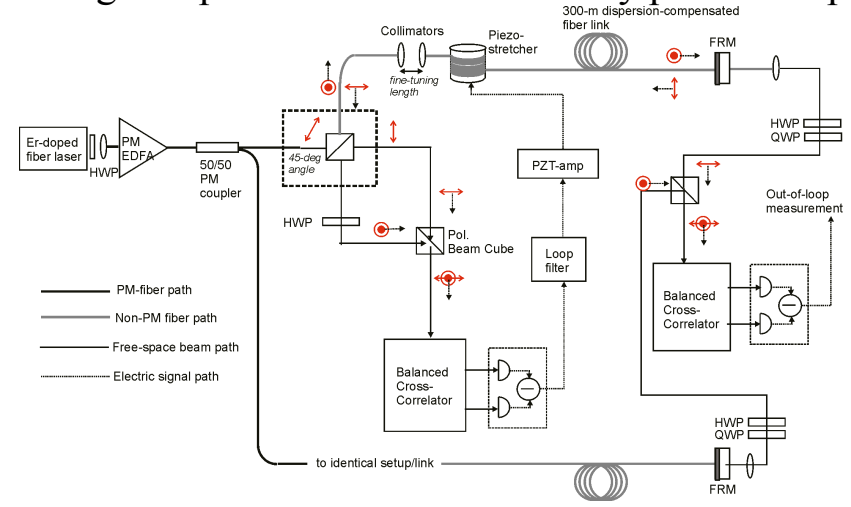


Figure 4.8: Fiber optic distribution system for FERMI. Only two links are shown in a configuration to measure the relative timing jitter between the two links in a test setup currently under construction at MIT.

The back reflected pulse train passes the input polarizing beam splitter and is cross correlated with the remaining pulse train from the modelocked laser in a balanced crosscorrelator. The cross-correlator is described later because it is currently intended for a patent application. The cross correlation signal is used to stabilize the length of the fiber via the nano-positioning stage and the fiber stretcher. In the setup shown in Figure 8 the two pulsed output signals from the fiber link are cross correlated with respect to each other in an external cross correlator to monitor the relative link stability. Yet, the polarization of the pulse streams from the fiber links is not yet stabilized. However, there are commercial polarization aligners available, for example from Thorlabs. Most likely the polarization rotation is not fast and can be easily tracked by home built control loops and half wave and quarter wave plates.

The resolution of the cross correlator will depend on the pulse width used. Currently, we plane to use about $100 \mathrm{fs}$ pulses, which can be also used for seeding of amplifiers after proper re-amplification. Also, the repetition rate can be easily reduced by using a modulator at the output of the fiber laser.

The fiber optic transmission link is composed of two sections of fiber. The first section is the PowerForm ${ }^{\mathrm{TM}}$ dispersion compensating fiber (DCF) from Avanex Corporation; the second section is Coring ${ }^{\circledR}$ SMF28 ${ }^{\mathrm{TM}}$ standard telecom fiber (SMF28) from Corning Incorporated. The detailed information including the dispersion and nonlinearity of these two types of fiber is listed in Table 4.1 [1,2], and the dispersion parameters and GVD parameter plots against wavelength are shown in Figure 4.9.

\begin{tabular}{|c|c|c|c|}
\hline & 2@1550nm & 3@1550nm & \\
\hline & $\mathrm{ps}^{2} / \mathrm{Km}$ & $\mathrm{ps}^{3} / \mathrm{Km}$ & $\left(\mathrm{W}^{*} \mathrm{Km}\right)^{-1}$ \\
\hline $\mathrm{DCF}$ & 154.7 & -0.91 & 5.3 \\
\hline SMF28 & -22.1 & +0.13 & 2.0 \\
\hline
\end{tabular}

Table 4.1: Fiber parameters: ${ }_{2}$ is the second order dispersion parameter, ${ }_{3} 3$ is the third order dispersion parameter, and, ${ }_{-}$is the nonlinear coefficient.

The DCF is manufactured by Avanex Corporation and commercially available. It can fully compensate the dispersion and dispersion slope of the standard telecom SMF28 fiber across the $1525 \mathrm{~nm}$ to $1565 \mathrm{~nm}$ pass band, and thus enabling the distortion free transmission of 100 fs pulses as long as the pulse energies used are not excessive. A fully dispersion compensated link system can be built utilizing these two types of fibers with proper fiber length configuration. As shown in Figure 4.10, according to the dispersion data, 1 unit length of DCF fiber fully compensates 7 unit length of SMF28 fiber. 


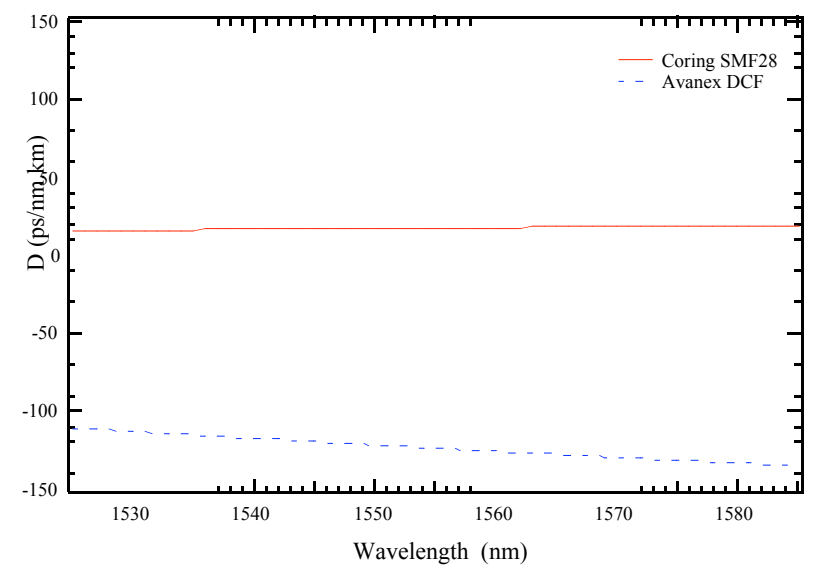

Figure 4.9: Dispersion parameters of SMF28 fiber and DCF fiber.

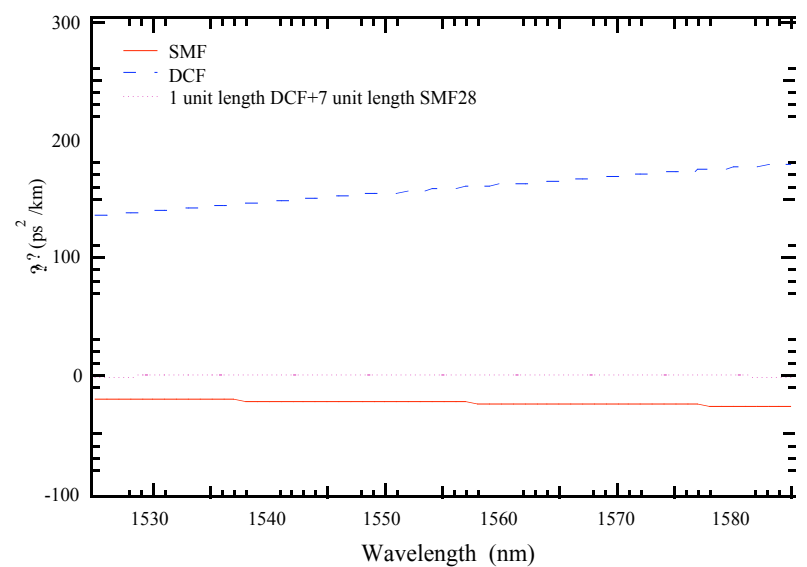

Figure 4.10: GVD parameters of SMF fiber and DCF fiber, and the sum of 1 unit length of DCF with 7 unit length of SMF28.

The proposed link configuration is shown in Figure 4.11. The first half of the link is 38 meters of the DCF (location A to B) and the second half is 266 meters of SMF28 (location $\mathrm{B}$ to $\mathrm{C}$ ). With this length configuration, the dispersion and dispersion slop will be fully compensated.

Transform limited optical pulses with a pulse duration of $100 \mathrm{fs}$ and pulse energy up to $50 \mathrm{pJ}$ enter the link at location A. The pulses acquire a positive chirp due to the positive GVD of the DCF. The pulse width will be stretched as the pulses propagate in the DCF fiber and eventually reach a maximum stretching of about $190 \mathrm{ps}$ at the end of the DCF section at location B. The pulses will then be compressed in the SMF28 section by its negative GVD, and the pulse width will decrease from 190 ps to its transfer limited pulse width around $100 \mathrm{fs}$ at the end of the link at location $\mathrm{C}$.

At the end of the link, location $\mathrm{C}$, half of the pulse energy will be reflected back to the link and eventually reach location A for cross correlation and link stabilization purposes. 
In this scheme, the pulses reach their minimum pulse width at the beginning and the end of the link (location A and C), so that an efficient optical cross-correlation can be performed at these two locations.

Note, $50 \mathrm{pJ}$ at $0.5 \mathrm{GHz}$ repetition rate corresponds to an average power of $25 \mathrm{~mW}$. Thus, the requested $20 \mathrm{~mW}$ of optical power, where $10 \mathrm{~mW}$ are used at the fiber end for cross correlation, is easy to transmit. In fact the simulation is a worst case scenario since the simulation in the return pass used the full $25 \mathrm{~mW}$ of power. Thus with respect to optical power there is enough room for parameter variations and in the final system for power variation which does not impact at all the system performance due to the balanced detector concepts that are tolerant against substantial power variations at both ends of the link. The final system achieved can be made tolerant to optical power variations and drifts of as much as 6-10dB.

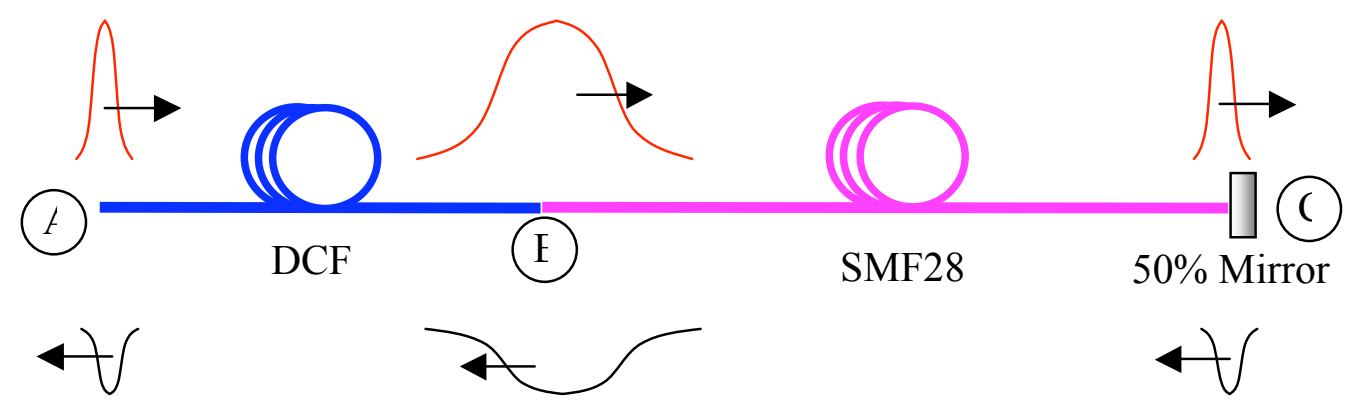

Figure 4.11: Link configuration. Blue section: 38 meters of DCF, Purple section: 266 meters of SMF28

The maximum pulse energy that can be transmitted is limited by soliton effects in the SMF28 fiber. Optical pulses may start to experience distortion at the end of the SMF28 fiber around location $\mathrm{C}$, if the pulse energy is higher than the soliton energy supported by the SMF fiber. Further increase of the pulse energy will result in pulse breaking, hence multiple pulses. Thorough numerical simulations have been carried out by Jeff Chen, based on these considerations the range of appropriate pulse energies is found.

In figure 4.12, normalized pulse power before and after the roundtrip are plotted in a time frame moving with the group velocity of the pulse. Pulses with a pulse energy less than $85 \mathrm{pJ}$ can be transmitted safely in the link without significant distortion. 


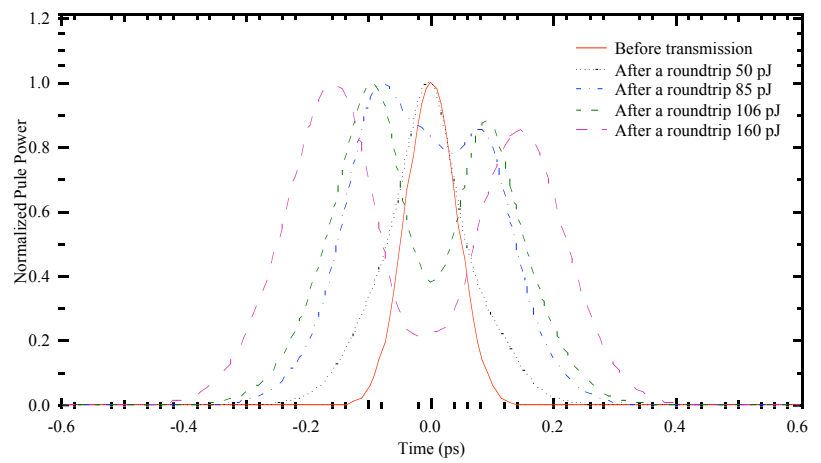

Figure 4.12: Normalized pulse shape before and after the transmission round trip for various pulse energies.

A pulse energy of $50 \mathrm{pJ}$ and a pulse duration of $100 \mathrm{fs}$ results in a pulse peak power of $500 \mathrm{~W}$. This is large enough to perform cross correlations in a frequency doubling crystal. Typical conversion efficiencies at the peak of the pulses of about $10^{-3}$ in typical crystals like $\mathrm{BBO}$ can be expected. At a repetition rate of $500 \mathrm{MHz}, 50 \mathrm{pJ}$ pulse energy corresponds to an average power of . At the mentioned conversion efficiency of $10^{-3}$ this would lead to enough signal power to reach sub-femtosecond timing resolution in the cross-correlator at shot noise limited detection, which can be reached in this case.

\subsection{OPTICAL MASTER OSCILLATOR}

The most promising candidates for ultra-low jitter optical master oscillators are $\mathrm{Er} / \mathrm{Yb}$ glass lasers [12], passively mode-locked Er-doped fiber lasers [13] and Yb-doped fiber lasers [14]. Er/Yb-glass lasers with sub-20 fs timing jitter have been demonstrated recently [12]. The crucial performance indicator for such a source is the phase noise or timing jitter integrated from $\mathrm{c} /(2 \mathrm{~nL})$ to the Nyquist frequency, i.e. half the repetition rate of the laser. This jitter will set an inherent limitation to the precision with which the pulse train can be distributed in the facility. Note, this is not necessarily a limitation for the RFdistribution, if the flywheel effect of the VCO in the RF-recovery according to Fig. 4 is properly exploited. In the final application, the OMO will follow within a certain bandwidth $f_{R O}$ tightly the RMO. If the bandwidth of the fiber link stabilization $f_{L L}$ is greater than $f_{R O}$ and the bandwidth of the lock for the RF extraction $f_{L R F}$ is less than $f_{R O}$, than the phase noise of the finally extracted RF is identical to the phase noise of the RMO for frequencies less than $f_{L R F}$ and identical to the phase noise of the VCO for frequencies beyond $f_{L R F}$. Then even high frequency fiber length fluctuations outside of $f_{L R F}$ are suppressed. Nevertheless, a very low jitter OMO is indispensable for femtosecond timing distribution.

Figure 4.13 (a) and (b) show the typical setup of fiber lasers and Er/Yb-glass bulk lasers. The fiber lasers are mode-locked by nonlinear polarization rotation and operate in the stretched pulse mode [15]. Due to the minimum length required for strong polarization rotation and the other fiber components, the repetition rate of fiber lasers is at maximum on the order of a few 

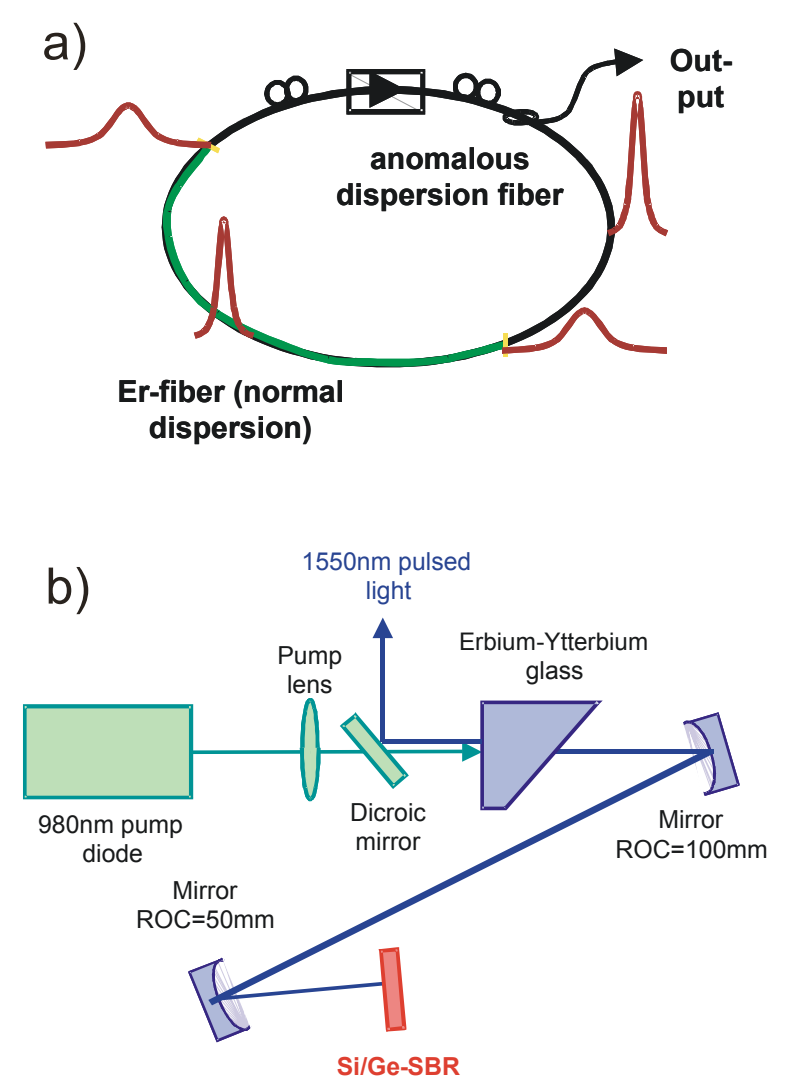

Fig. 4.13: Schematic of (a) a stretched pulse Er-fiber laser mode-locked by nonlinear polarization rotation and (b) a Er/Yb-glass laser mode-locked by a semiconductor saturable absorber mirror.

hundred MHz. In contrast the Er/Yb-glass bulk laser is mode-locked by a semiconductor saturable absorber [16]. The length of the gain medium can be as short as a few millimeter and, therefore, if necessary these lasers can be scaled to tenth of $\mathrm{GHz}$ in repetition rate [17].

We have constructed stably operating sub-picosecond Yb-fiber, Er-fiber and bulk $\mathrm{Er} / \mathrm{Yb}$-glass lasers and evaluated the timing jitter of the different sources. Figure 7 shows the timing jitter for the $\mathrm{Er} / \mathrm{Yb}$ and $\mathrm{Yb}$-fiber lasers and for comparison the phase noise of a high quality Marconi microwave oscillator often used in accelerator facilities is shown.

Fig. 4.14 shows the Single-Sideband (SSB) Phase Noise of an Yb-fiber laser, Erfiber laser and $\mathrm{Er} / \mathrm{Yb}$-glass laser measured with an Agilent 5052a signal source analyzer. The fiber lasers had a repetition rate of $36 \mathrm{MHz}$ and the Er/Yb-glass laser of $216 \mathrm{MHz}$. The measurements were carried out on the corresponding harmonic at $1.3 \mathrm{GHz}$. The integrated timing jitter from $1 \mathrm{kHz}$ to the respective Nyquist bandwidths, i.e., half of the laser repetition rate, is measured to be about $18 \mathrm{fs}$ and $10 \mathrm{fs}$ for the YDFL and EDFL, respectively. For comparison, the phase noise of a very low noise frequency generator, a Marconi 2041, is also plotted. As the lasers are free running, the performance of the microwave oscillator is slightly superior in the low frequency regime $(<10 \mathrm{kHz})$, but at frequencies of $100 \mathrm{kHz}$, the mode locked lasers reach a comparable level of stability, 
with the EDFL having the lowest noise among the three at frequencies higher than 20 $\mathrm{kHz}$. Both lasers would be already suitable for an overall sub-100 fs timing distribution system, which is an important next step to achieve in several FEL facilities. On the long run, the EDFL seems to be a stronger candidate for a master oscillator due to the availability of a larger variety of components at $1550 \mathrm{~nm}$, and perhaps more importantly, transmission fibers with both signs of dispersion, which allow the construction of dispersion-compensated fiber links. The measured timing jitter for both lasers is substantially higher than the noise limit given by the spontaneous emission noise. Several effects in the photodetection process lead to significant distortion of the actual phase noise spectrum. Due to the filtering of a single harmonic of the laser repetition rate, the power level of this harmonic is on the order of $-20 \mathrm{dBm}$, even when saturating a typical photodetector. The thermal noise floor of the $50 \mathrm{Ohm}$ resistor used to terminate the photodiode is at $-178 \mathrm{dBm}$. That results in a minimum noise floor of $-158 \mathrm{dBc}$ for the single sideband phase noise. The phase noise measurement device employed in this study (Agilent 5052) implements a correlation technique, which provides an additional noise suppression of up to $10 \mathrm{~dB}$. Even with this improvement, the phase noise of the reference oscillator itself constitutes another measurement limit. Another effect, which plagues phase noise measurements, is amplitude to phase conversion in the photodiode [14]. The EDFL and YDFL have extremely low intensity fluctuations, nevertheless amplitude to phase conversion may contribute as much as 5 fs additional timing jitter.

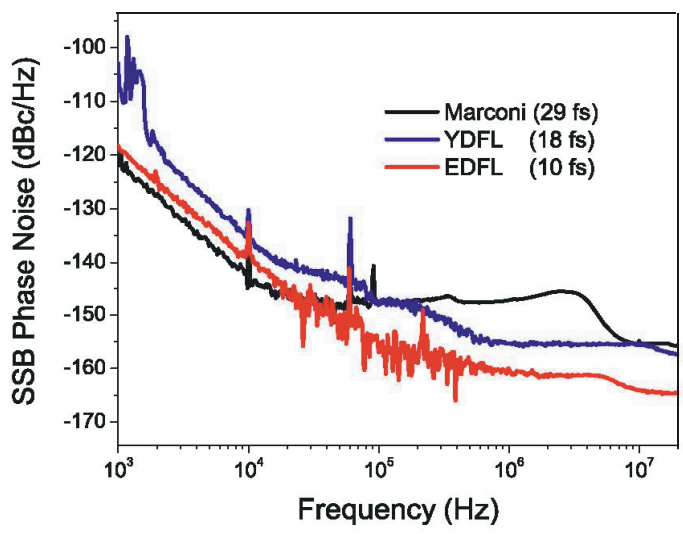

Fig. 4.14: Single-Sideband Phase noise (SSB) of a Marconi microwave oscillator and those of a Ytterbium doped fiber laser at $1 \mu \mathrm{m}$ and an Erbium doped fiber laser at $1.5 \mu \mathrm{m}$

This stretched pulse mode-locked Yb-fiber laser generates the shortest pulses and operates close to the zero average dispersion point of the cavity. In that case, frequency fluctuations can not couple to timing fluctuations, which is known as the Gordon-Haus jitter. The timing jitter also scales proportional to the pulse width of the generated pulses. Both facts contribute to the low timing jitter of this $\mathrm{Yb}$-laser [18,19]. This operation principle will be also applied in the next version of the Er-fiber laser.

The use of fiber lasers is a natural choice for implementing an optical master oscillator, because of the ease of coupling to the fiber distribution system. However, 
should higher repetition rate lasers become necessary for optimum timing distribution, the properly packaged Er/Yb-glass lasers can be made equally stable.

\subsection{OPTICAL-TO-OPTICAL SYNCHRONIZATION}

Synchronization is necessary not only between optical and RF-subsystems but also between different optical systems, for example, the photo-injector laser and the master oscillator as shown in Fig. 4.1. Figure 4.15 shows how the optical-to-RF synchronization scheme based on timing transfer in the optical domain, discussed in section II, can be used to tightly synchronize two pulsed lasers with each other. By using the RFsynchronization module first the RF signal is extracted from one laser (ML-laser 1 in Fig. 4.15). This RF-signal drives the phase modulator of another RF-synchronization module. However, the error signal from balanced detector of the second loop drives a piezoelectric transducer (PZT) that controls the repetition rate of the second laser (MLlaser 2 in Fig. 4.15) rather than the VCO. In this way, an effective synchronization of multiple lasers can be achieved.

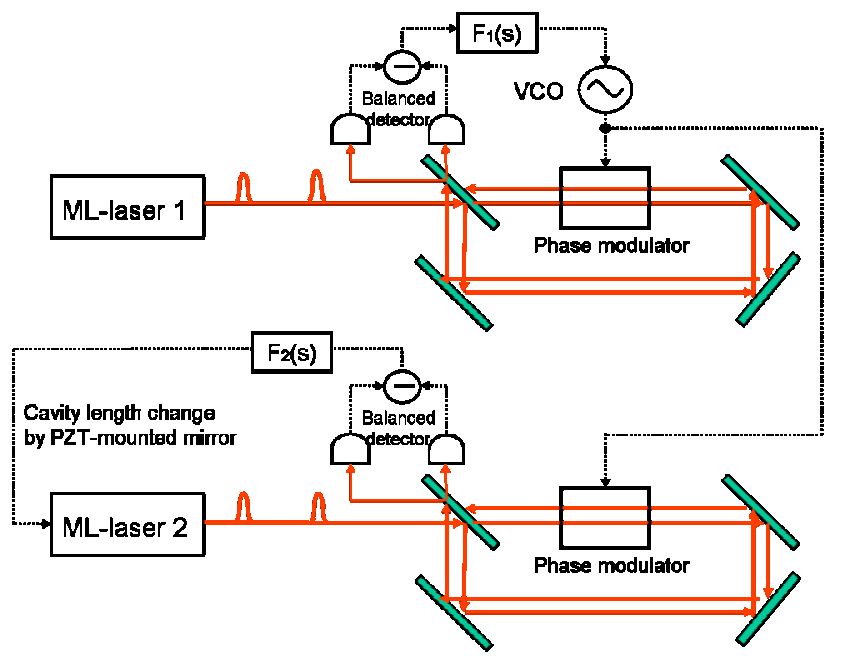

Fig. 4.15: Optical-to-optical synchronization scheme with the based on RF-synchronization techniques.

Another method for optical-to-optical synchronization is based on balanced optical cross correlation. This method enabled so far the most accurate synchronization of multiple laser sources [10]. In addition to these techniques one can envision that the distributed optical pulses can be directly used for injection locking of local laser systems or seed of amplifiers eventually after frequency doubling.

\section{REFERENCES}

[1] J. Kim, F. Ö. Ilday, F. X. Kärtner, O. D. Mücke, M. H. Perrott, W. H. Graves, D. E. Moncton and T. Zwart, "Large Scale Timing Distribution and RF-Synchronization for FEL-Facilities," FELConference 2004, Trieste, Italy, Aug. 26-29, 2004.

[2] Poseidon Scientific, http://www.psi.com.au.

[3] R. Holzwarth, Menlo-Systems, Priv. Comm..

[4] E. N. Ivanov, S. A. Diddams and L. Hollberg, IEEE J. Sel. Top. Quant. Elec. 9, 1059 (2003).

[5] A. Bartels, S. A. Diddams, C. W. Oates, G. Wilpers, J. C. Bergquist, W. H. Oskay, and L. Hollberg, Opt. Lett. 30, 667 (2005).

[6] J. Kim, F. X. Kärtner, and M. H. Perrott, Opt. Lett. 29, 2076 (2004). 
[7] J. Ye, J.-L. Peng, R. J. Jones, K. W. Holman, J. L. Hall, D. J. Jones, S. A. Diddams, J. Kitching, S. Bize, J. C. Bergquist, L. W. Hollberg, L. Robertsson, L.-S. Ma, J. Opt. Soc. Am. B 20, 1459 (2003).

[8] K. W. Holman, D. J. Jones, D. D. Hudson, J. Ye, Opt. Lett. 29, 1554 (2004).

[9] K. W. Holman, D. D. Hudson, D. J. Jones, J. Ye, Opt. Lett. 30, 1225 (2005).

[10] T. R. Schibli, J. Kim, O. Kuzucu, J. T. Gopinath, S. N. Tandon, G. S. Petrich, L. A. Kolodziejski, J. G. Fujimoto, E. P. Ippen, F. X. Kärtner, Opt. Lett. 28, 947 (2003).

[11] A. Winter, B. Schmueser, H. Schlarb, F. Ö. Ilday, J.-W. Kim, J. F. X. Kärtner, "High-precision optical synchronization systems for x-ray free-electron lasers.," In Proceedings of FEL 2005, Stanford University, CA.

[12] J. B. Schlager, B. E. Callicoatt, R. P. Mirin, N. A. Sanford, D. J. Jones, J. Ye, Opt. Lett. 28, 2411 (2003).

[13] G. Lenz, K. Tamura, H. A. Haus, E. P. Ippen, Opt. Lett. 20, 1289 (1995).

[14] F. Ö. Ilday, J. R. Buckley, H. Lim, F. W. Wise, W. G. Clark, Opt. Lett. 28, 1365 (2003).

[15] K. Tamura, E. P. Ippen, H. A. Haus, and L. E. Nelson, Opt. Lett. 18, 1080-1082 (1993).

[16] F. J. Grawert, S. Akiyama, J. T. Gopinath, F. Ö. Ilday, H. Shen, J. Liu, K. Wada, L. C. Kimerling, E. P. Ippen and F. X. Kärtner, Opt. Lett. 30, 329-331 (2005).

[17] S. C. Zeller, L. Krainer, G. J. Spuehler, R. Paschotta, M. Golling, D. Ebling, K. J. Weingarten, and U. Keller, 30th European Conference on Optical Communication. ECOC 2004, 2004, pt. 4, p 918-19 vol.4.

[18] S. Namiki, and H. A. Haus, IEEE J. Quantum Electron. 33, 649-659 (1997).

[19] C. X. Yu, S. Namiki, and H. A. Haus, IEEE J. Quantum. Electron. 33, 660-668 (1997).

\section{Synchronization technology development at LBL}

\subsection{RF transmission}

In our scheme, RF phase information is transmitted by amplitude modulating a $\mathrm{CW}$ laser signal, and receiving the signal on a photodiode. The RF power available from the receiver photodiode is determined by the saturation and damage thresholds of the diode, and by the stimulated Brillouin scattering (SBS) threshold of the fiber. For a $300 \mathrm{~m}$ fiber with typical core diameter of 9 microns, the CW power limit is about $40 \mathrm{~mW}$. Amplitude modulation increases the threshold by breaking the CW signal into three lines, the carrier and sidebands. The sideband separation is wider than the SBS bandwidth (a few hundred $\mathrm{MHz}$ ), so each line would have to independently exceed the SBS threshold. Depending on the modulation format, the carrier can be larger or smaller than the sidebands, or equal in power. The suppression of the carrier in the proposed external modulation scheme can be controlled by varying the bias voltage on the amplitude modulator. This is a DC term that is added to the RF, moving the center of the sinusoidal modulation back and forth on the $\sin ^{\wedge} 2$ transfer function of the modulator. By adjusting the bias, it is possible to equalize the carrier and sideband powers as shown in the graph below, as measured on an optical spectrum analyzer.

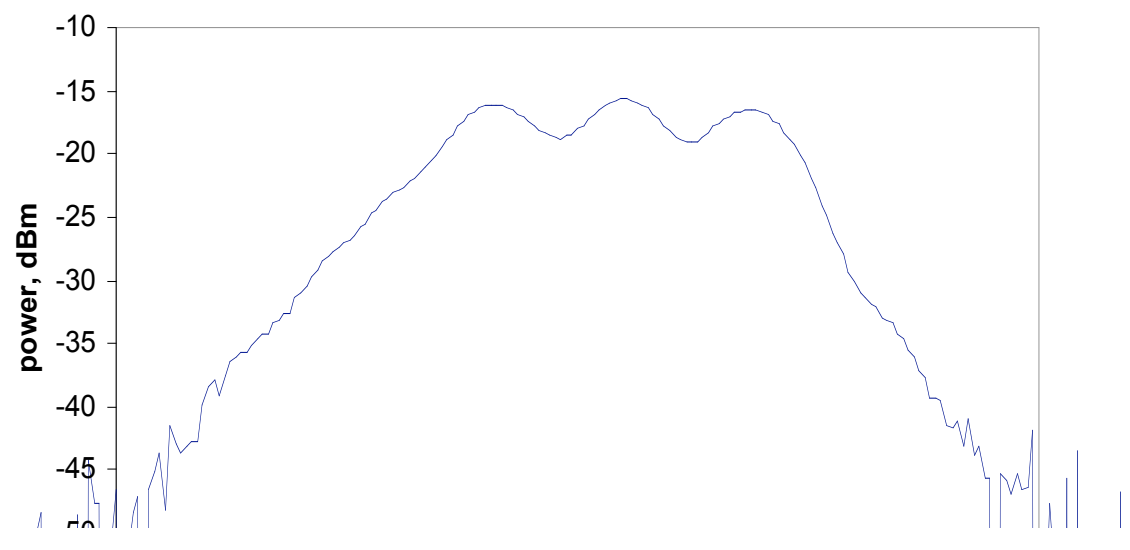


Figure 5.1. Optical power in the carrier and sidebands at $3 \mathrm{GHz}$ amplitude modulation frequency, when bias is adjusted to make them equal.

This shows that the SBS threshold can be increased by a factor of 3 due to modulation. It is important to avoid seeding the SBS with a sideband at the Stokes shift, however, as this would decrease the threshold markedly. If the sidebands were separated from the carrier or from each other by about $11 \mathrm{GHz}$, an upper sideband would create SBS gain and the retroreflected carrier or the other sideband would see the gain, causing most of the launched power to be diffracted backwards. The modulator generates odd or even harmonics depending on the bias, so the potential for seeded SBS still exists, and should be closely monitored when signals are introduced into long fibers.

A photodiode driven near saturation can exhibit amplitude-to-phase conversion, resulting in timing jitter created by amplitude instability (RIN noise in the CW laser, for instance). Some photodiodes have higher saturation power levels, resulting in better performance in an RF-over-fiber link. We tested a commercial 12GHz photoreceiver (Microwave Photonic Systems) using a network analyzer. The analyzer supplied $3 \mathrm{GHz}$ RF to an amplifier which drove an amplitude modulator, modulating light from a $1570 \mathrm{~nm} \mathrm{CW}$ laser. RF modulated on the optical signal was received on the photodiode and returned to the network analyzer, which measured RF phase as the optical power was varied. Results are shown in the graph below, expressed as shift in time rather than phase. The slope is $67 \mathrm{fs} / \mathrm{mW}$. To achieve e.g. $<20 \mathrm{fs}$ stability, the power on the diode would have to be stable to within $0.4 \mathrm{~mW}$ or $10 \%$. The proposed system has little polarization dependent loss (PDL), so variations in polarization will not have a large effect on the power received by the diode. Optical power regulation to better than 5\% can be achieved by feeding back to the RF power applied to the fiber frequency shifter (explained below).

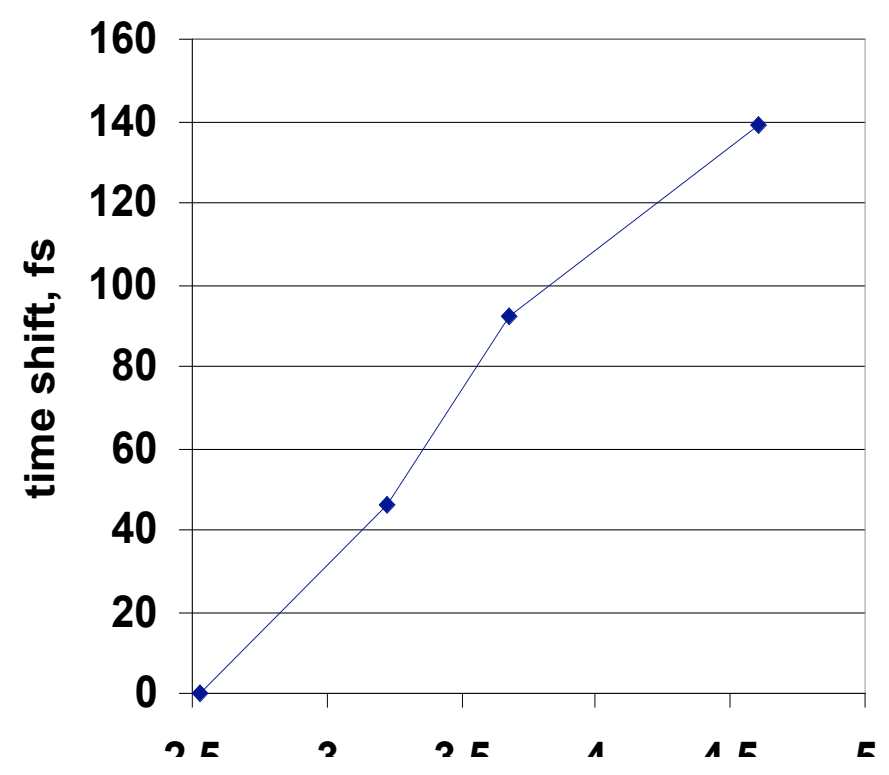


Figure 5.2. Variation in temporal delay of a $3 \mathrm{GHz}$ signal through a photodiode versus average optical power.

A demonstration of low phase noise RF transmission using amplitude modulated $\mathrm{CW}$ light has been made using a commercial RF-over-fiber link consisting of a transmitter and receiver connected by a short fiber, with no fiber length drift compensation. A low noise RF source was constructed by detecting the pulse train of a modelocked laser with a photodiode, bandpass filtering at $2 \mathrm{GHz}$ and amplifying in a low noise amplifier. The integrated jitter of the source from $1 \mathrm{kHz}$ to $40 \mathrm{MHz}$ was $11 \mathrm{fs} \mathrm{RMS}$, limited by the photodiode shot noise at around $-150 \mathrm{dBc}$. This RF signal was input to the link transmitter and the received signal was observed, with an integrated jitter of $15 \mathrm{fs}$ RMS from $1 \mathrm{kHz}$ to $40 \mathrm{MHz}$, as shown in the figure below. No additional phase noise added by the link could be observed from $10 \mathrm{~Hz}$ to $10 \mathrm{kHz}$. RF power loss through the link is about $20 \mathrm{~dB}$, with the input to the link at $5 \mathrm{dBm}$ and its output at $-16 \mathrm{dBm}$. Differences in signal levels into the noise analyser may account for the link output noise being lower than the input noise at some frequencies, an apparent experimental error. Minimizing acoustic noise in the lab improved the measurement by tens of femtoseconds, indicating this sensitive measurement needs to be refined. Nevertheless, the conclusion is that amplitude modulated $\mathrm{CW}$ laser signals received by a photodiode are viable for timing transmission systems with less than $100 \mathrm{fs}$ integrated jitter.

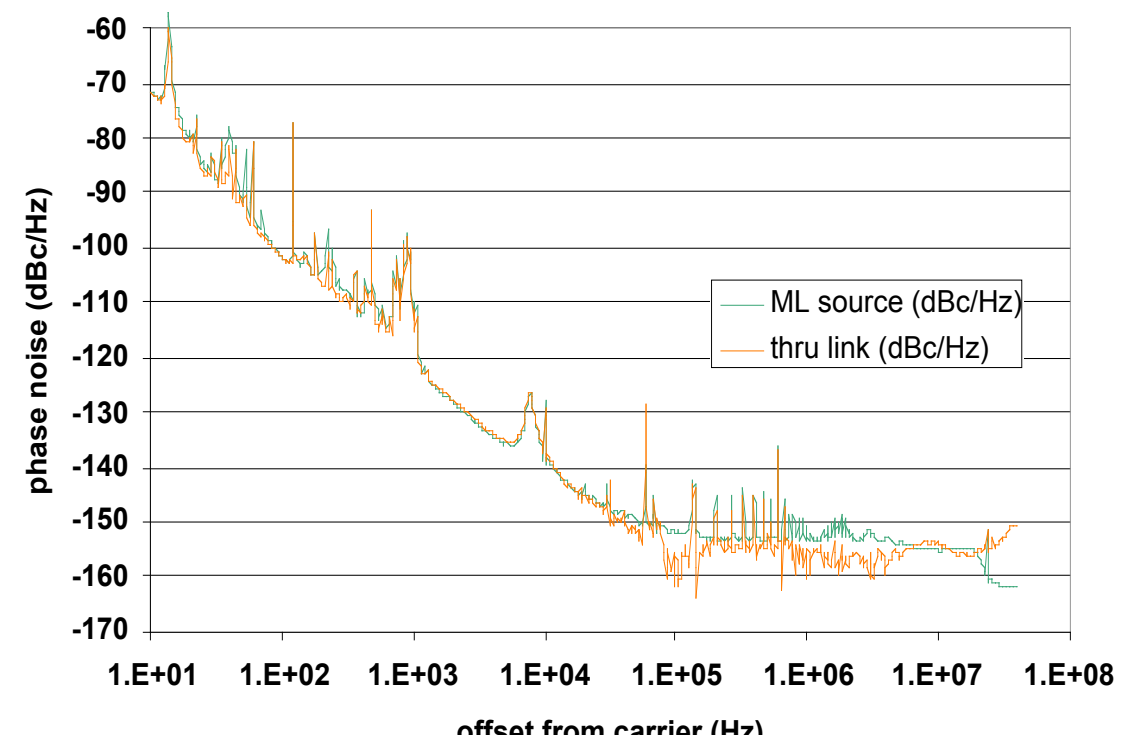

Figure 5.6. Phase noise of $2 \mathrm{GHz}$ source (green curve) and signal transmitted through a modulated $\mathrm{CW}$ fiber optical link (orange curve). 


\subsection{Line stabilization via interferometer}

In this $\mathrm{CW}$ transmission scheme, stabilization of the transmission fiber delay is via an unequal-arm Michelson interferometer. Figure 1 shows the optical and electronic block diagram.

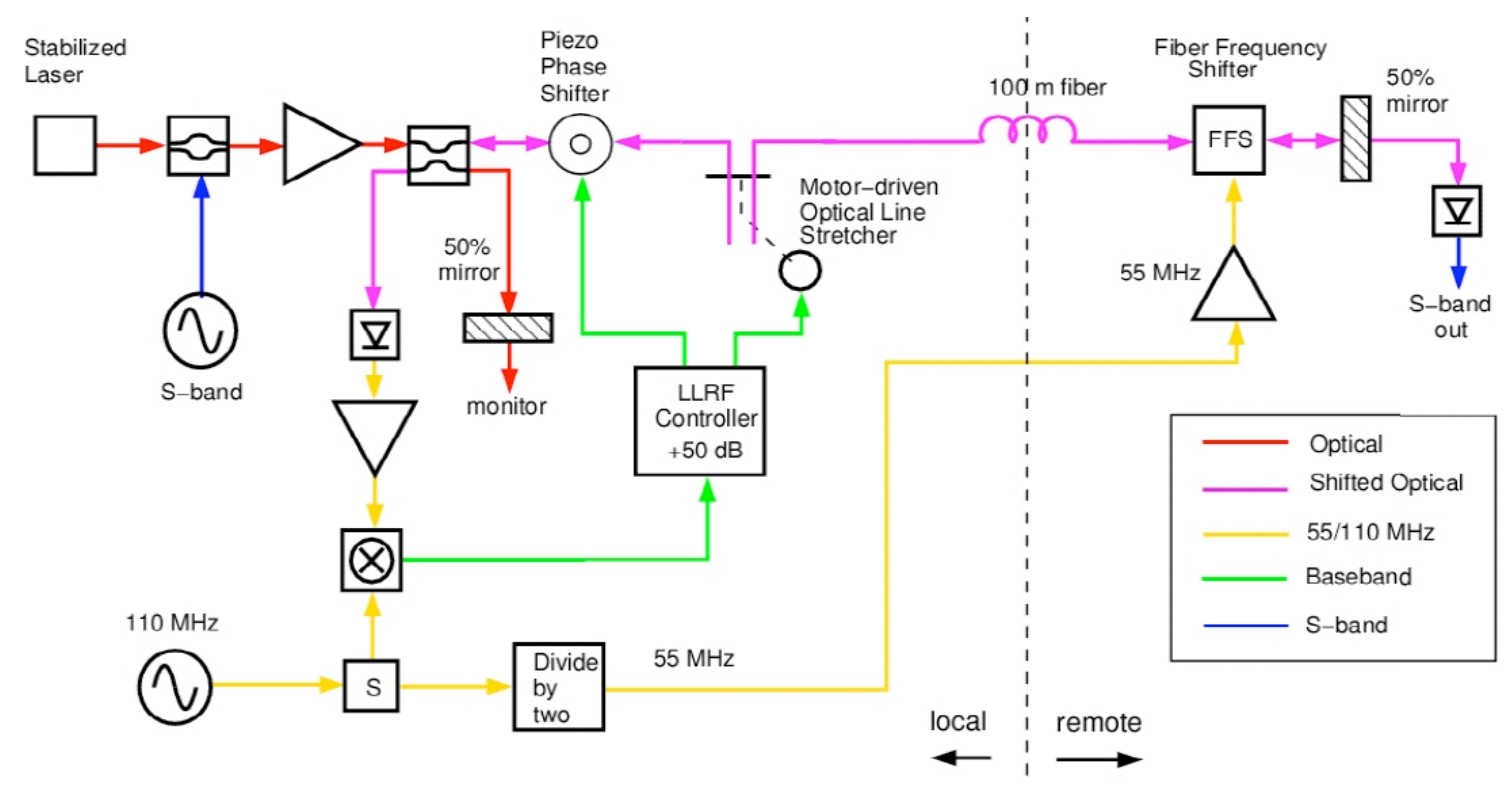

Figure 5.3. Frequency shifting Michelson interferometer fiber length stabilization scheme.

The CW laser mentioned above is wavelength-stabilized by side locking to an absorption line in acetylene, to a stability of $10^{\wedge}-8$. This translates to an instability of $15 \mathrm{fs}$ over a $300 \mathrm{~m}$ fiber. The $\mathrm{CW}$ laser signal is introduced into an unequal arm Michelson interferometer, consisting of the transmission fiber (long arm) and a reference fiber (short arm). At the receiver end of the fiber an acousto-optic frequency shifter doubly shifts the retroreflected optical carrier (and AM sidebands) by 50MHz. When the long and short arm signals are superimposed on a photodiode, a beat at $100 \mathrm{MHz}$ is seen, which can be compared in phase with a $100 \mathrm{MHz}$ reference oscillator. When these $100 \mathrm{MHz}$ signals are stabilized in relative phase, the optical phase delay of the transmission fiber is constant with respect to the reference. Control of the delay through the transmission fiber is achieved by a piezoelectrically controlled fiber spool (for fast perturbations) and a motorized optical delay (for slow drifts). All fibers in the interferometer not stabilized electronically are stabilized in temperature to 0.01 degree $\mathrm{C}$.

We check the operation of the control loop via another interferometer, in this case a Mach-Zehnder using the stabilized long fiber as one arm, and a temperature controlled fiber as the other. The figure below shows a simplified block diagram. The monitor channel does the same phase comparison as the control channel, providing us an out-ofloop measurement with high precision. 


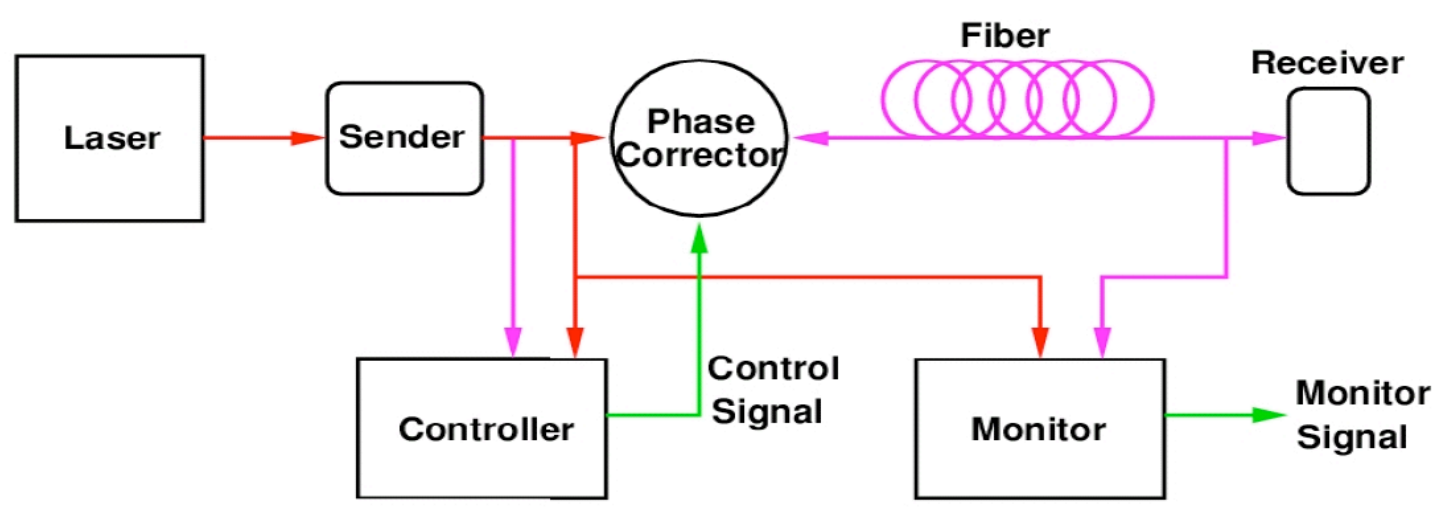

Figure 5.4. Out-of-loop monitoring of Michelson stabilizer using a Mach-Zehnder interferometer in parallel.

Experiments have shown this scheme can stabilize a $100 \mathrm{~m}$ fiber to within a few femtoseconds over many hours. An example plot below shows results of stabilizing a $100 \mathrm{~m}$ fiber strung around the walls of our lab, to pick up low frequency acoustics due to air flow. The phase comparison from the monitor channel (Mach-Zehnder interferometer) was made using a digital phase detector (an up/down counter with 128pi range). The drift rate is $0.13 \mathrm{fs}$ per hour, with a superimposed sinusoidal variation of $1 \mathrm{fs}$ peak-to-peak due to air conditioning cycling.

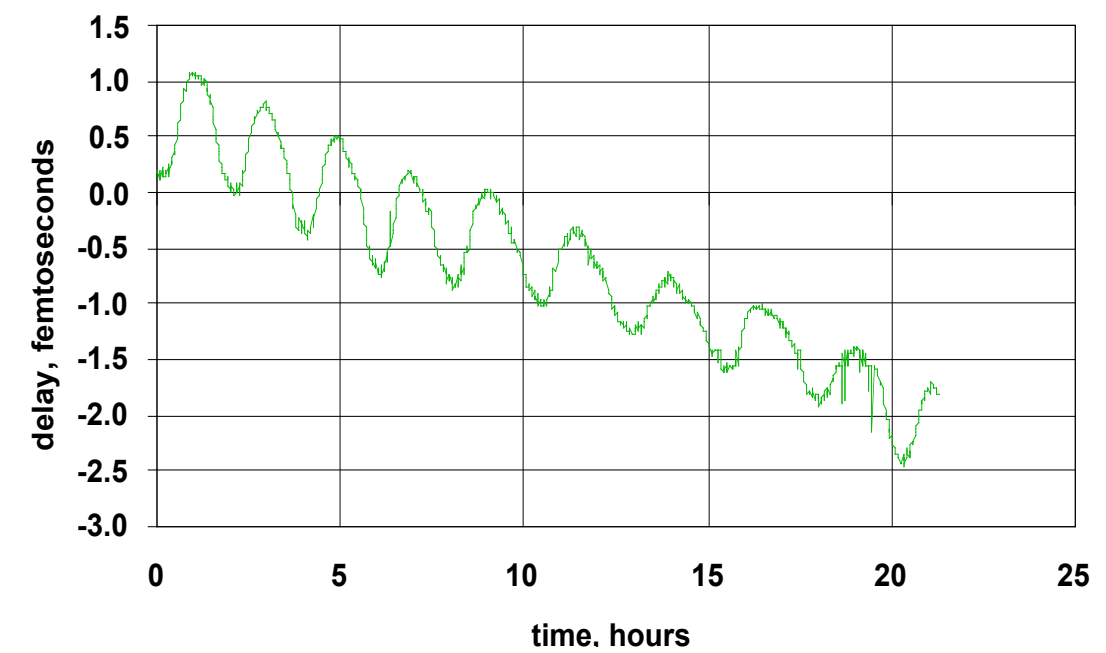

Figure 5.5. Delay variation of a stabilized fiber, measured using a second interferometer.

In another experiment, we built two interferometrically stabilized lines, using both of them in a Mach-Zehnder arrangement. Each line was stabilized using an unequal-arm Michelson scheme described above, using light from the same laser. The two frequency shifters apply a shift of opposite sign, so that the optical wave is upshifted by $55 \mathrm{MHz}$ in one arm and downshifted by $55 \mathrm{MHz}$ in the other. Light transmitted through the two arms is combined in a directional coupler and the beat between the two waves observed on a 
photodiode. Thus we can observe relative phase shifts between two interferometrically stabilized fibers. A block diagram is shown below.

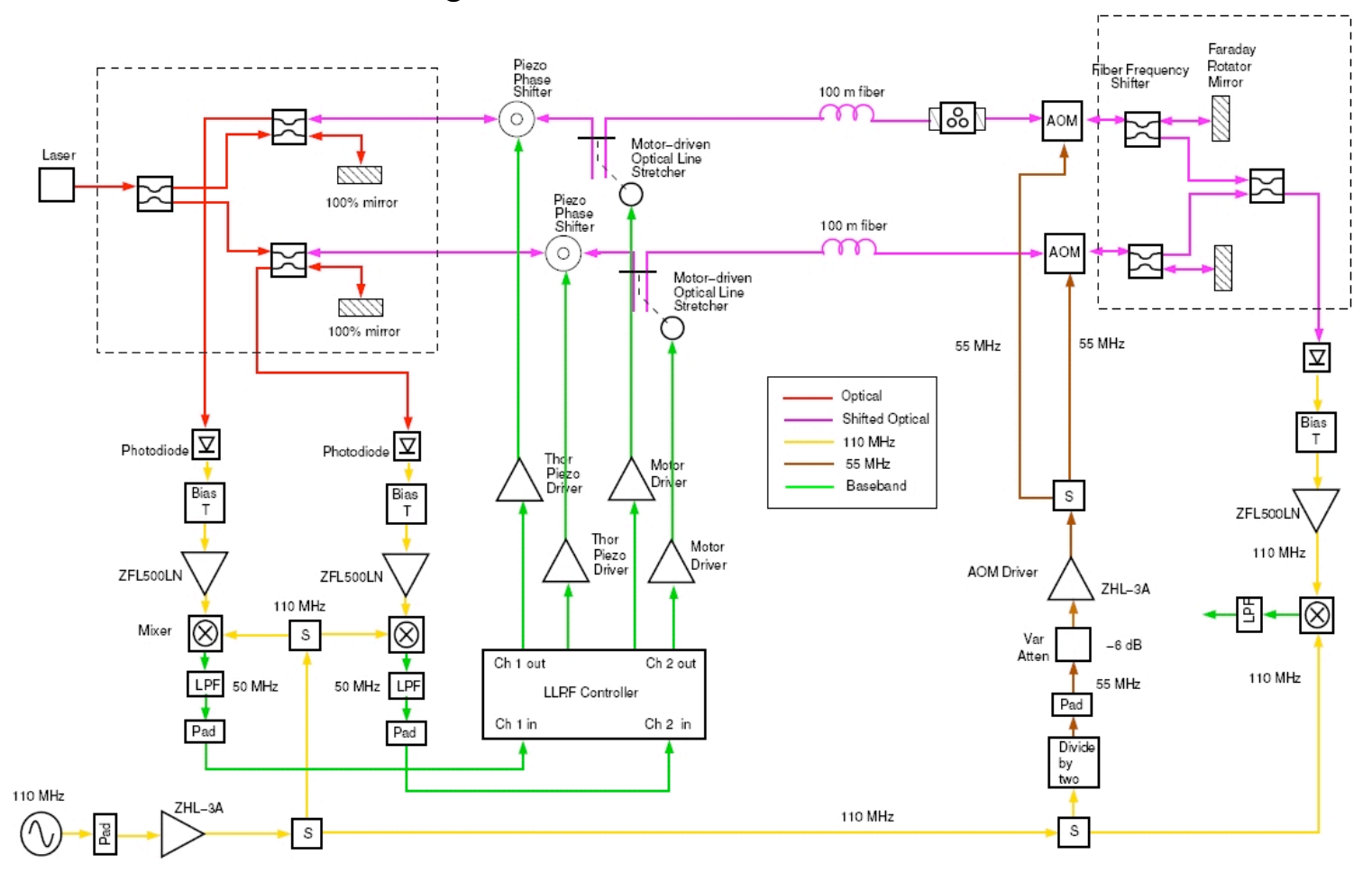

Figure 5.6. Tandem stabilized line experiment.

With $100 \mathrm{~m}$ of fiber in each stabilized line, the relative phase between the Mach-Zehnder output and a reference oscillator was recorded using a vector voltmeter. Data from a 35 hour run is shown below.

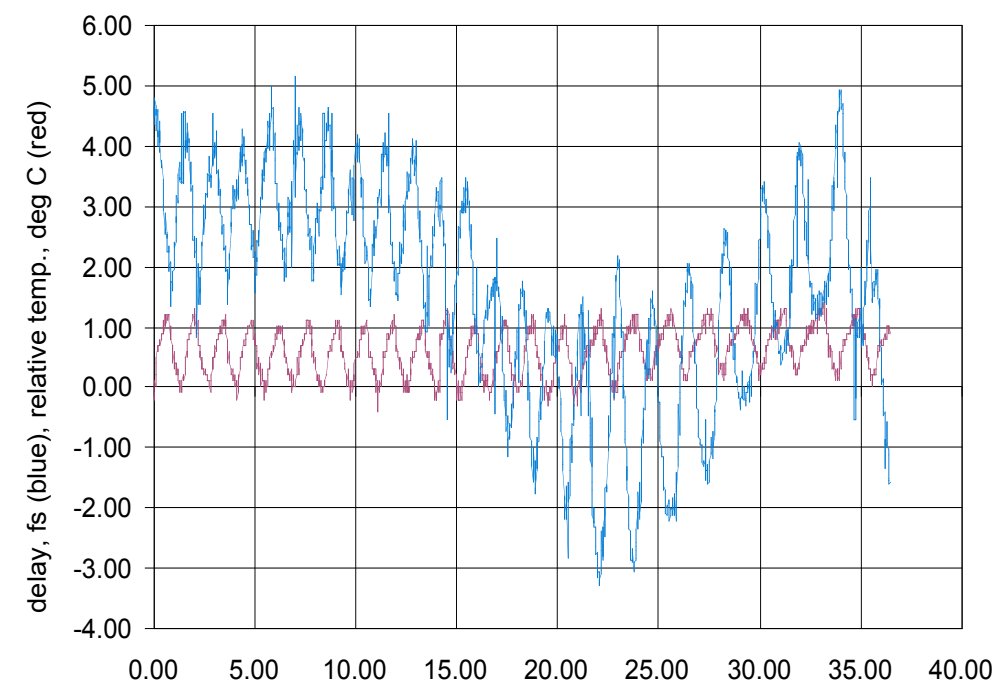

Figure 5.7. Relative temporal drift of two $100 \mathrm{~m}$ lines (blue), compared with temperature (red). Vertical scale is in femtoseconds, horizontal in hours. 
There is about $3 \mathrm{fs} p-p$ drift due to temperature cycling. The larger temperature-induced variations in this experiment may be due to a different heat sinking scheme for the temperature-stabilized boxes that contain the Michelson interferometer reference arms. It may also be that the previous experiment underestimated the true temperature drift, as the reference arm for the Mach-Zehnder monitor interferometer was contained in the same box as the Michelson reference arm, and they could drift together. The more recent tandem line experiment is a better indicator of system performance. The longer term variation seems to be diurnal, possibly due to changes in humidity, which we have reduced in earlier experiments by dehumidifying the reference arm box.

Though we precisely stabilize the optical phase delay using an interferometer, the group delay is what is important for AM transmission. The group or phase delay in the fiber will be the fiber length divided by the speed of light times the group or phase refractive index. The phase and group indices are related according to the following equation, which must include dispersion:

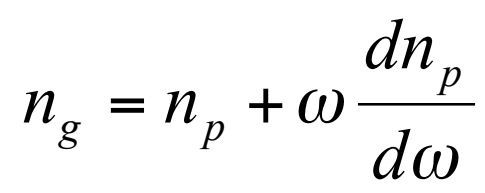

If all the terms in the above equation had the same temperature coefficient, or if dispersion was constant with temperature, a constant correction could be added to the group or phase delay to compensate delay variation with temperature. In fact dispersion has a different temperature coefficient, which makes compensation more complicated. Over a small enough range, the difference in temperature compensation for group or phase delay can be approximated by a constant multiplication factor which can be found by measurement of the fiber. Measurements made of several different fibers by others $(1,2)$ allowed us to estimate that the temperature coefficients of group and phase index differ by a few percent. In one case, the difference was around $2 \%$. For a $200 \mathrm{~m}$ fiber, there is $7 \mathrm{ps}$ per degree $\mathrm{C}$ of phase delay drift. If group delay was uncompensated, the delay error could be $140 \mathrm{fs}$ per degree.

This implies a possible compensation scheme. If the total phase delay correction to the interferometer is known, the variation in phase index is also known. An additional correction is then applied to compensate the group delay variation instead. The group delay is thus controlled by measuring changes in the phase delay. All the computations can be done by the digital controller. If the compensation scheme is able to reduce the error by a factor of ten, errors due to group versus phase index can be reduced to less than $20 \mathrm{fs}$ per degree. If the fiber is routed in the linac tunnel, the temperature excursions will be within $\sim 1$ degree due to the temperature stability requirements of the other equipment. 


\subsection{Short pulse laser synchronization}

Lasers can be remotely synchronized using techniques derived from recent "frequency comb" techniques. The basic concept is to compare and control two spectral lines of two pulsed lasers in order to synchronize their pulse trains. Figure 1 shows the frequency comb lines of two pulsed lasers, with two of the frequencies of one laser compared with two nearby frequencies of the other. By small adjustments of the second laser, its frequencies can be matched in phase with the first, thus synchronizing the two pulse trains in time.

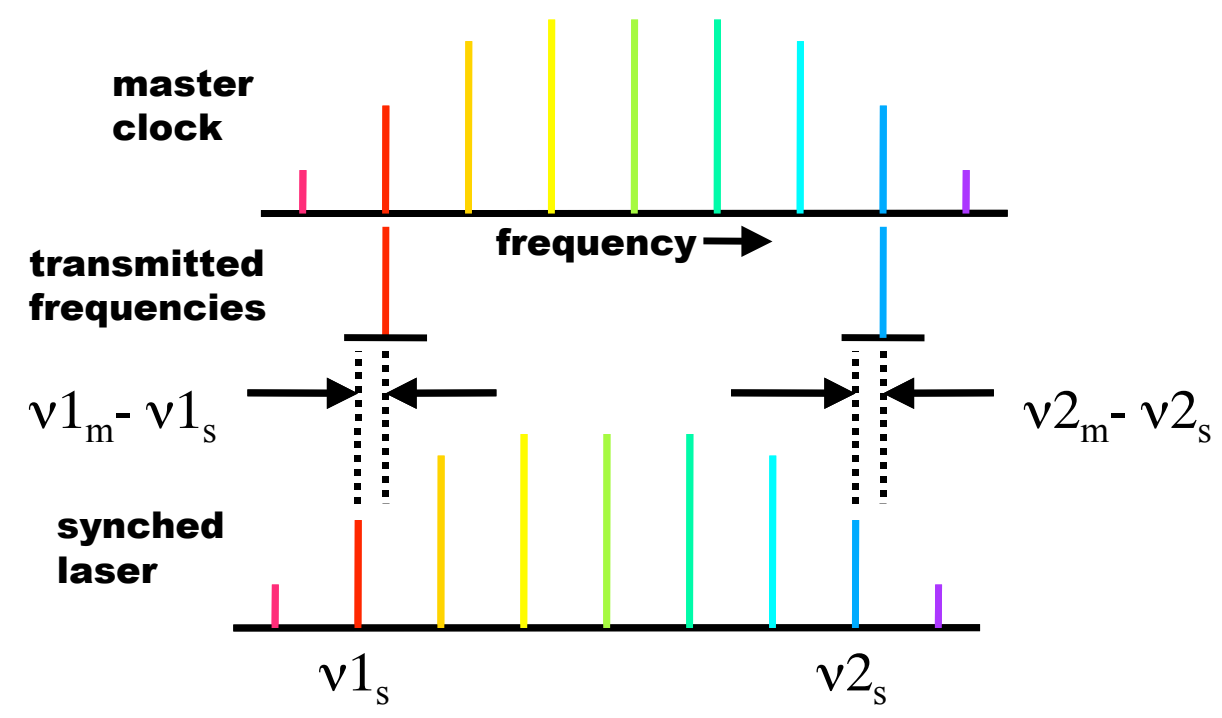

Figure 5.8. Comparison between two modelocked laser frequency combs in order to lock the laser repetition rates.

Referring to figure 5.7 above, the lasers are synchronized by comparing pairs of comb lines of the two lasers, $\mathrm{n} 1 \mathrm{~m}-\mathrm{n} 1 \mathrm{~s}$ and $\mathrm{n} 2 \mathrm{~m}-\mathrm{n} 2 \mathrm{~s}$. The differences are turned into error signals and used to control the synched laser. It is also possible to see this scheme as a comparison between two teraHertz frequencies, $n 1 m-n 2 m$ versus $n 1 s-n 2 s$. Looked at this way, it is clear that the sensitivity to differences in timing between the lasers can be very high, even if the phase sensitivity is modest. If the two comb lines are $5 \mathrm{THz}$ apart (spanning the telecom C-band, which is easily done with a fiber laser), one is comparing the phase of two $5 \mathrm{THz}$ frequencies. If the phase sensitivity is a modest 1 degree, the temporal sensitivity is $0.6 \mathrm{fs}$. Using the bandwidth available in an optical comb provides the "leverage" needed to achieve precise synchronization. This principle has been used by other researchers to demonstrate synchronization of two lasers to a few femtoseconds $(3,4)$. In the work of Bartels (3), the synchronized lasers must be carrier-envelope offset (CEO) stabilized, which puts extra constraints on the laser, and may preclude the use of a laser more appropriate to the applications in Fermi. The proposed two-frequency optical scheme avoids the use of CEO stabilized lasers, while potentially achieving the same level of synchronization. Note that in the two cases in references 3 and 4 the two schemes operate at very different frequencies (14GHz vs $456 \mathrm{THz})$, but demonstrate about the 
same level of synchronization. This indicates that the limits to these schemes are factors other than just the temporal versus phase sensitivity. At this level of temporal precision, many factors could affect the timing, requiring careful engineering beyond the basic laboratory proof-of-principle.

Two optical frequencies for synchronizing pulsed lasers are derived from a pulsed master clock laser, which is an excellent source of phase-synchronized optical signals. Two frequencies are derived from the master clock by locking two slave CW lasers. One of the $\mathrm{CW}$ lasers serves as an absolute frequency reference, locking one of the master lasers to that single frequency, while another $\mathrm{CW}$ laser is locked to another of the master laser's comb lines. Alternatively, two slave single frequency $\mathrm{CW}$ lasers can be locked via interferometers to two of the master laser's comb lines.

Locking of the optical frequencies of two lasers is accomplished using an optical phase locked loop. Light from both the clock and the CW laser is interfered on a photodiode, and the difference frequency is observed as an RF signal. The phase of this signal is compared with an RF reference and a DC signal is derived that controls the optical frequency of the clock or the slave laser.

Once two frequencies that represent the timing information are generated, they are transmitted through an interferometrically stabilized fiber. Because of the difference in temperature coefficients between the phase and group velocities, mentioned above, it is advantageous in this case to use two interferometrically stabilized fibers, so that each fiber stabilizes the phase of a single frequency $\mathrm{CW}$ optical signal and no issues related to dispersion arise. Thus the fiber interferometers are stabilizing exactly the information which is being used, that is the optical phase.

Once transmitted, the two frequencies are received by interferometers connected to the laser to be synchronized. This process is essentially the reverse of the transmitting process. Each of the two frequencies is interfered with light from the synchronized pulsed laser, yielding the difference frequency and phase. This is used to control the frequencies of the pulsed laser and thus its repetition rate. In this way the synchronized laser is made to follow the timing of the clock laser. 


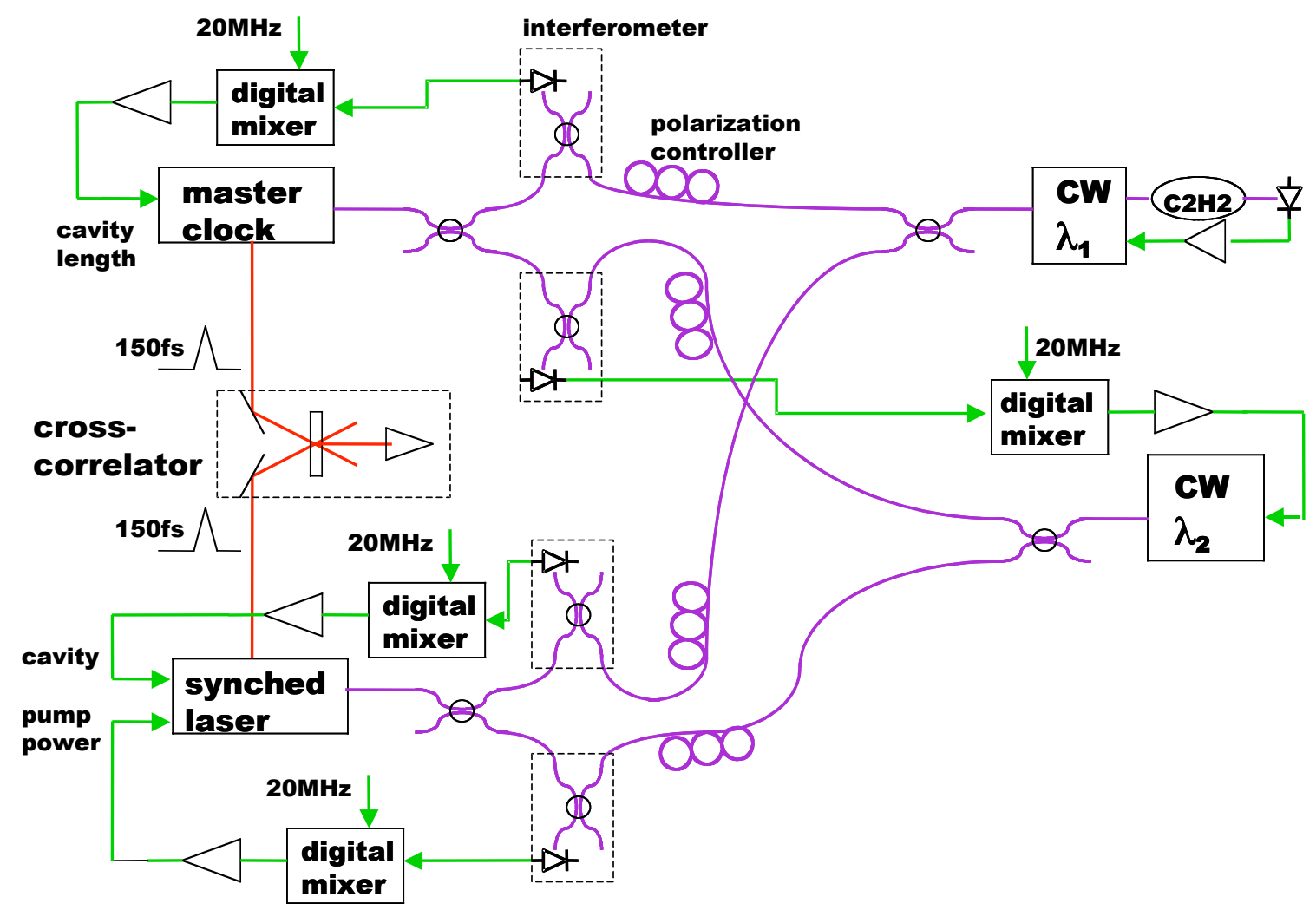

Figure 5.9. Experimental layout of two-frequency synchronization of two modelocked fiber lasers. Cross-correlator is used to measure synchronization.

Performance of this system can be estimated by observing the noise in error signals of the various control loops. At present, loop gain is limited by the onset of oscillation because none of the loop filters are optimized. We have measured the stability of two kinds of optical phase locked loops; a CW laser locked to a comb line and a modelocked laser comb line locked to a $\mathrm{CW}$ laser. In each configuration the error signal was observed on an oscilloscope and FFT. In both cases, the performance is about the same, represented by the power spectrum shown below when a modelocked laser was locked to a $\mathrm{CW}$. The RMS instability is about $63 \mathrm{fs}$, mainly at frequencies at resonant peaks of the piezoelectrically controlled frequency shifters in the CW and modelocked lasers. When gain is increased, oscillation starts at these frequencies. 


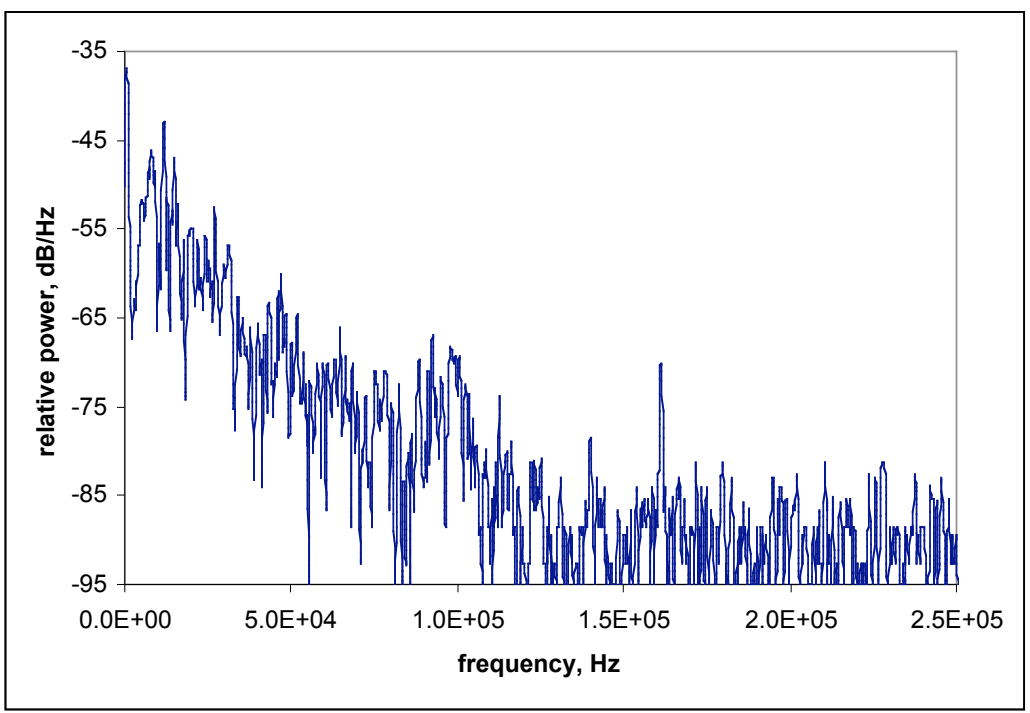

Figure 5.10. Loop error signal spectrum for modelocked laser locked to $\mathrm{CW}$ laser.

In order to improve the loop gain, we have been working with a laser company (Menlo Systems) to improve performance of the piezo cavity mirror mount. They were able to remove numerous resonances, as indicated by interferometric measurements shown below.

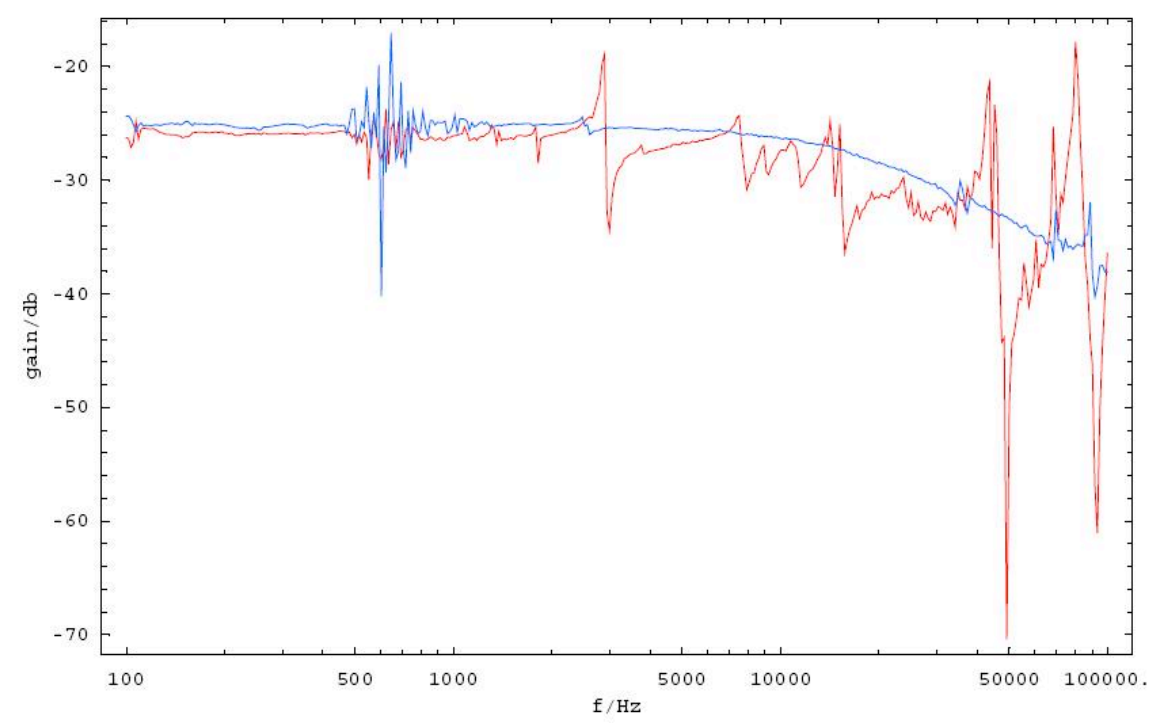

Figure 5.11. Frequency response of old (red curve) and new (blue curve) piezo mirror mounts for commercial modelocked fiber laser.

A refurbished laser was delivered, and we measured the amplitude and phase transfer function of the new mount. A swept signal from a network analyzer was sent to the piezo amplifier, and the laser's $2 \mathrm{GHz}$ harmonic observed on a photodiode was input to an FM demodulator, then returned to the network analyzer. Thus we could translate an audio range signal into an RF frequency modulation via the laser, and demodulate to retrieve the audio signal. This data will be analyzed and incorporated into a numerical model of 
the control loop, so that a special, optimized loop filter can be designed. This approach was used on the fiber delay stabilizer, and was quite successful, so we expect significant improvement over the simple PI loop filter. Also, the vendor of the CW laser (Koheras) has announced an improved mechanical design which will likely reduce the resonances in the laser frequency control. We will purchase another of these lasers and test its frequency modulation transfer function within the next year.

There are four optical phase locked loops in the two-frequency synch scheme, which include additional uncorrelated (by initial assumption) noise. If the requirement for system stability is 10fs RMS, this may be budgeted to the transmission system and the laser synch system. Transmission stability will be exclusively phase stability, as there are two lines which deliver pure phase information on unmodulated CW carriers. Both lines will experience the same temperature perturbations if they are routed together, so the slow errors are correlated, and the net transmission error will be about $2 \mathrm{fs}$ peak-to-peak, based on previous measurements. The errors due to noise in the PLLs are assumed divided equally in quadrature between them, so that each one would have to have about 5 fs RMS error. This is 12 times less than the currently measured error with the old piezo mirrors in the laser. Thus an increase in gain of 12 is needed to achieve a system stability of $10 \mathrm{fs}$. Based on our experience with the fiber stabilization control loop, this seems a modest improvement.

The system implementation of the two-frequency laser synch scheme is shown below. The first figure shows the transmitter and line delay stabilizer. In this case the CW laser locked to an absolute reference is the master laser. Since the modelocked laser reprate is also stabilized to an RF reference, the other optical comb lines will be accurately referenced to the absolute standard. This feature may be useful if wavelength references are needed in the facility.

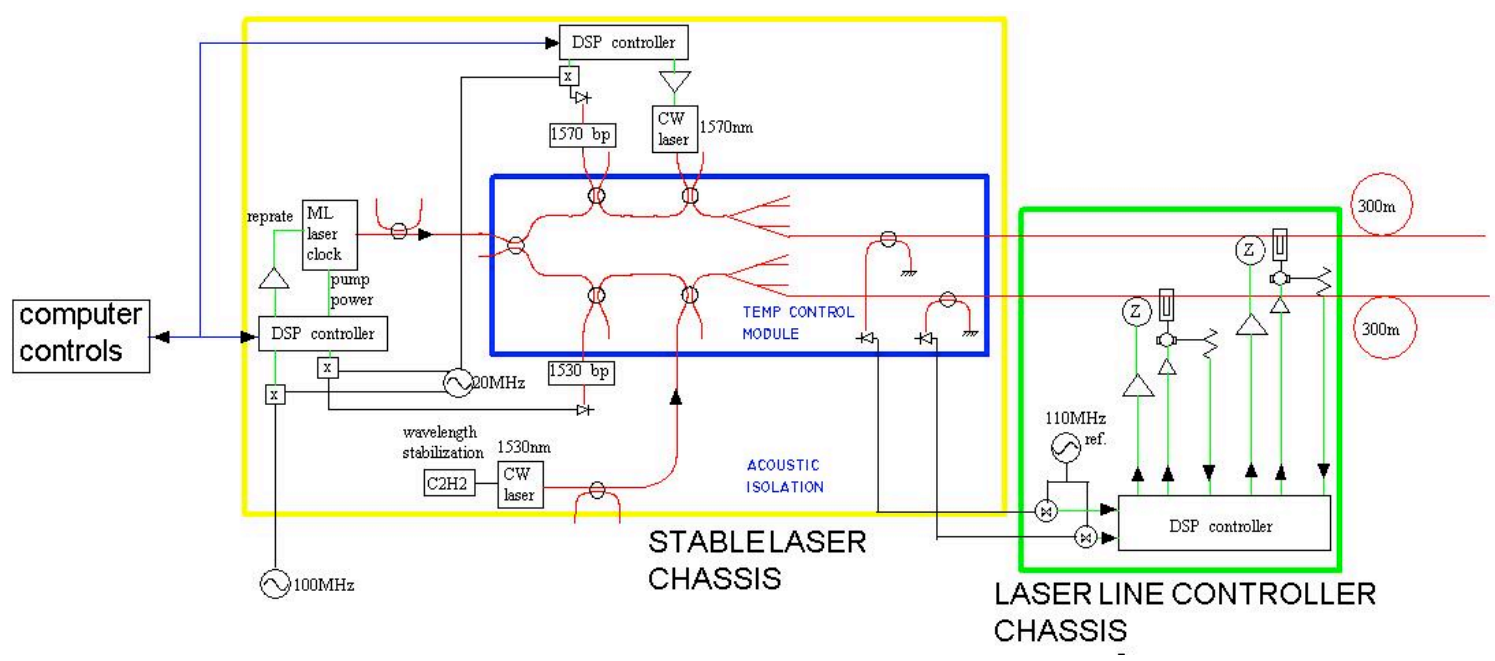

Figure 5.12. Two-frequency laser synch transmitter and line controller.

The laser synch receiver in the case shown below is a $1550 \mathrm{~nm}$ modelocked fiber laser, although other types of lasers can also be used. Each received optical signal must be 
polarization controlled to be stable linear polarization before being interfered with the synched laser comb lines (in PM fiber components). A polarization controller linearizes the polarization before part of the signal is retroreflected by the $50 \%$ mirror. This scheme has the advantage that polarization mode dispersion is also corrected by the line stabilizer. When a Faraday rotator mirror is used, as in the RF transmission scheme, the optical wave returns to the transmitter along the orthogonal axis of the slightly birefringent fiber. Any change in delay due to a change in birefringence is averaged out. When the linearized wave is retroreflected, it travels along the same birefringent fiber axis, so that the signal back to the line controller contains information about the actual path of the wave, not the average of its path and the orthogonal polarization one.

A coarse lock is provided by a $100 \mathrm{MHz}$ (or the exact master clock reprate) reference frequency, so that the right comb line is chosen. The two optical frequencies sent are $5 \mathrm{THz}$ apart, which is 50,000 times the repetition rate. Thus if the synched laser reprate is one part in $5 \times 10^{\wedge} 4$ different from the clock, it will still lock but not be synchronized. The coarse lock must provide a reference that is accurate to $100 \mathrm{MHz} / 5 \mathrm{e} 4$ or $2 \mathrm{kHz}$. When the reference and the synched laser reprates are beat against each other, they will be able to lock correctly if the difference frequency is less than $2 \mathrm{kHz}$. The lock controller will adjust for this condition before locking the laser optically.

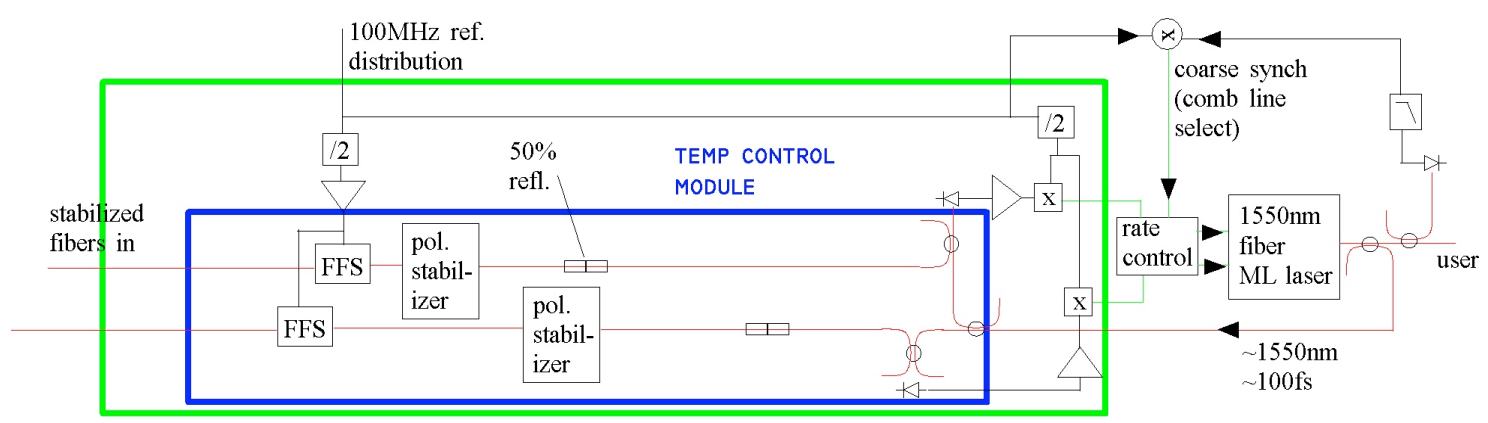

Figure 5.13. Two-frequency synchronized fiber laser.

\subsection{References}

1. T. Kato and Y. Koyano, "Temperature dependence of chromatic dispersion in various types of optical fiber," Opt. Lett. 25, p. 1156 (2000).

2. K. S. Kim and M. E. Lines, "Temperature dependence of chromatic dispersion in dispersion-shifted fibers: Experiment and analysis,” J. Appl. Phys. 73, p. 2069 (1993).

3. A. Bartels, S. A. Diddams, T. M. Ramond, and L. Holberg, "Mode-locked Laser Pulse Trains with Subfemtosecond Timing Jitter Synchronized to an Optical Reference Oscillator", Opt. Lett. 28, p. 663 (2003). 
4. 1. R. K. Shelton, S. M. Foreman, L-S Ma, J. L. Hall, H. C. Kapteyn, M. M. Murnane, M. Notcutt and J. Ye, "Subfemtosecond Timing Jitter Between Two Independent, Actively Mode-locked Lasers”, Opt. Lett. 27, p. 312 (2002). 